\title{
On the Layering Transition of an SOS Surface Interacting with a Wall. II. The Glauber Dynamics
}

\author{
Filippo Cesi ${ }^{1}$, Fabio Martinelli ${ }^{2}$ \\ ${ }^{1}$ Dipartimento di Fisica, Università "La Sapienza", P.le A. Moro 2, 00185 Roma, Italy. \\ e-mail: cesi@vaxrom.roma1.infn.it \\ ${ }^{2}$ Dipartimento di Matematica, III Università, Via C. Segre 2, 00146 Roma, Italy. \\ e-mail: martin@mat.uniroma3.it
}

Received: 19 December 1994/in revised form: 19 May 1995

\begin{abstract}
We continue our study of the statistical mechanics of a 2D surface above a fixed wall and attracted towards it by means of a very weak positive magnetic field $h$ in the solid on solid (SOS) approximation, when the inverse temperature $\beta$ is very large. In particular we consider a Glauber dynamics for the above model and study the rate of approach to equilibrium in a large cube with arbitrary boundary conditions. Using the results proved in the first paper of this series we show that for all $h \in\left(h_{k+1}^{*}, h_{k}^{*}\right)\left(\left\{h_{k}^{*}\right\}\right.$ being the critical values of the magnetic field found in the previous paper) the gap in the spectrum of the generator of the dynamics is bounded away from zero uniformly in the size of the box and in the boundary conditions. On the contrary, for $h=h_{k}^{*}$ and free boundary conditions, we show that the gap in a cube of side $L$ is bounded from above and from below by a negative exponential of $L$. Our results provide a strong indication that, contrary to what happens in two dimensions, for the three dimensional dynamical Ising model in a finite cube at low temperature and very small positive external field, with boundary conditions that are opposite to the field on one face of the cube and are absent (free) on the remaining faces, the rate of exponential convergence to equilibrium, which is positive in infinite volume, may go to zero exponentially fast in the side of the cube.
\end{abstract}

\section{Introduction}

This paper is the second part of a work, begun in [CM], about the equilibrium and non-equilibrium statistical mechanics of a SOS surface above a fixed wall at low temperature and attracted towards it by a very weak external field.

Although the problem is clearly relevant for the understanding of wetting phenomena (see e.g. [FP1, FP2]) and, more generally, for the study of two dimensional interfaces, our main motivation originates from the study of the ergodic

Work partially supported by grant SC1-CT91-0695 of the Commission of European Communities. 
properties of Glauber-type dynamics for discrete spins systems on the lattice when the thermodynamic parameters, e.g. the temperature and the magnetic field, are in the one phase region of the phase diagram.

An important open problem in this context can be formulated as follows: consider a discrete spin system with a unique Gibbs measure $\mu$ and assume in addition that

(*) the conditional expectation of a local function $f$ in a cube of side $L$ tends exponentially fast to its thermodynamic limit $\mu(f)$ when $L \rightarrow \infty$, uniformly in the boundary conditions (this is the case if, for example, the Dobrushin-Shlosman uniqueness condition holds [DS]).

Can one conclude that a reversible Glauber-like dynamics with respect to $\mu$ is ergodic and the approach to equilibrium is exponentially fast in the sup norm?

A positive answer to this problem has been given, as far as we know, only when, either

(1) the dynamics is attractive [MO1], or

(2) the lattice is two-dimensional [MOS].

Notice that condition $(*)$ has been proved for ferromagnetic Ising type models in arbitrary dimension, for any temperature above the critical one or for small temperature and arbitrary external field different from zero [MO1], and in the 2D Ising model in the whole one phase region [SSh].

In the general (non-attractive) case, exponential convergence to equilibrium has been proved only with the additional assumption that

$(* *)$ the gap in the spectrum of the generator of the dynamics in a finite region is bounded away from zero uniformly in both the boundary conditions and the size of the region.

We refer the reader to [SZ] and references therein and to [MO1, MO2, LY], where only cubic regions were considered.

It is important to stress that $(* *)$ is equivalent to the condition that the truncated correlation functions in a finite volume (e.g. a cube) decay exponentially fast everywhere inside the volume (in the bulk and close to the boundary) so that, in particular, it implies $(*)$.

The contribution of [MOS] consisted in showing that in two dimensions (**) is actually equivalent to $(*)$. The argument of [MOS] heavily depends on the facts that the boundary of a two dimensional square is one dimensional, and so it breaks down in higher dimensions, where $(* *)$ has been proved only deep inside the one phase region (see for more details [MO1]). Thus no "finite volume results" are available near the boundary of the one phase region, particularly near a line of a first order phase transition.

Already in [MO1] (see also [MOS]) the possibility was discussed that, even if the thermodynamic parameters correspond to a unique phase and $(*)$ holds, in a finite cube one could have some sort of "boundary phase transition" which could slow down dramatically the approach to equilibrium.

The main goal of this paper is to provide substantial evidence that this phenomenon happens in the 3D Ising model at low temperature and nonzero magnetic field. More precisely we will argue that it is possible to tune the magnetic field as a function of the inverse temperature $\beta$ in such a way that the gap in the spectrum of the generator of the dynamics in a finite cube with suitably chosen boundary 
conditions becomes exponentially small in the side of the cube. Notice that for the same value of the thermodynamic parameters and thanks to the result of [MO1], the gap of the infinite volume dynamics as well as the thermodynamic limit of the gap in finite cubes with + boundary conditions [S1] are bounded away from zero.

In order to clarify the discussion, let us consider the 3D Ising model in a cube $Q$ of side $\mathrm{L}$, at low temperature and small, positive external field $h$. As boundary conditions we take -1 on the bottom face $V$ of the cube and free (i.e. absent) on the other faces. Since the magnetic field is positive, the typical configurations of the systems will be mostly pluses (plus phase) away from the bottom face. Thus there will be, with large probability, a unique contour $\Gamma$ separating the plus bulk phase from the minus boundary conditions on the bottom face, and it is quite clear that the statistical properties of such a contour will play an important role in the mixing properties of the Gibbs measure of the system and on the rate of approach to equilibrium for an associated Glauber dynamics.

In order to attack the problem one can simplify the model by approximating the contour $\Gamma$ with a two dimensional surface $\varphi=\{\varphi(x)\}_{x \in V}$, where the random variable $\varphi(x)$ represents the height of the surface at $x$ above the bottom face of the cube, and one may assume that the probability distribution of the surface is that of the solid on solid model (SOS),

$$
\begin{aligned}
\mu_{V}^{\psi}(\varphi)=\frac{1}{Z^{\psi}(V)} \exp & -\frac{\beta}{2} \sum_{\substack{x, y \in V ; \\
|x-y|=1}}|\varphi(x)-\varphi(y)|-\beta h \sum_{x \in V} \varphi(x) \\
& \left.-\sum_{\substack{x \in V \\
|x-y|=V^{c}}}|\varphi(x)-\psi(y)|\right] .
\end{aligned}
$$

A kinetic version of the model is readily obtained by considering a Glauber dynamics for it, namely a single spin Markov process on the configuration space, reversible with respect to $\mu_{V}^{\psi}(\varphi)$ and such that each move consists in replacing at some site $x, \varphi(x)$ with $\varphi(x) \pm 1$.

The equilibrium problem for this system has been recently studied in [DM] and further analyzed in [CM]. We recall for the reader's convenience the main result of [CM], since it plays an essential role in our analysis of the non-equilibrium case.

Theorem [CM]. There exists $\beta_{0}$ such that for all $\beta \geqq \beta_{0}$ there are positive numbers

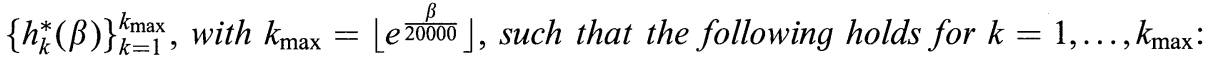

(i) $\frac{1}{4} e^{-4 \beta k} \leqq \beta h_{k}^{*}(\beta) \leqq 4 e^{-4 \beta k}$.

(ii) If $h_{k}^{*}(\beta)<h<h_{k-1}^{*}(\beta)$ (define $h_{0}^{*}(\beta)=+\infty$ ), then

(a) there exists a unique Gibbs measure for the interaction (0.1),

(b) there exist $m(\beta, h)>0, C(\beta, h)>0$ such that for any $N \geqq\lfloor 8 / h+1\rfloor$

$$
\sup _{\psi, \psi^{\prime} \in \Omega}\left|E_{Q_{N}}^{h, \psi} \varphi(0)-E_{Q_{N}}^{h, \psi^{\prime}} \varphi(0)\right| \leqq C(\beta, h) e^{-m(\beta, h) N}
$$

where $E_{Q_{N}}^{h, \psi}(\varphi(0))$ denotes the expected value of the height of the surface at $x=0$ in a square $Q_{N}$ of side $N$ and center at the origin, with boundary conditions $\psi$. 
(iii) If $h=h_{k}^{*}(\beta)$, then both partitions functions $Z^{k}\left(Q_{N}\right)$ and $Z^{k+1}\left(Q_{N}\right)$, with boundary conditions $\psi \equiv k$ and $\psi \equiv k+1$ respectively, admit a convergent cluster expansion. Hence there are at least two distinct extreme Gibbs measures.

In the present paper we turn our attention to the Glauber dynamics of the SOS surface in a square $Q_{N}$ of side $N$, with boundary conditions $\psi$ and magnetic field $h$; more precisely we analyze the gap ${ }^{h, \psi}\left(Q_{N}\right)$ in the spectrum of its generator which is related to the relaxation time to equilibrium. Our main result is

Theorem. In the same setting as in Theorem $[C M]$ we have, for all $k=1, \ldots, k_{\max }$,

(i) if $h_{k}^{*}(\beta)<h<h_{k-1}^{*}(\beta)$ then there exist $L_{0}(\beta, h), \kappa(\beta, h)>0$ such that

$$
\inf _{L \geqq L_{0}} \inf _{\psi \in \Omega} \operatorname{gap}^{h, \psi}\left(Q_{L}\right) \geqq \kappa(\beta, h) .
$$

(ii) If $h=h_{k}^{*}(\beta)$, then there exist positive constants $C_{1}(\beta, h), C_{2}(\beta, h)$ such that for all $N>10 / h$

$$
C_{1}(\beta, h) e^{-100 \beta k N} \leqq \operatorname{gap}^{h, \emptyset}\left(Q_{N}\right) \leqq C_{2}(\beta, h) e^{-\frac{1}{40} \beta N},
$$

where $\emptyset$ means free boundary conditions.

Going back to the 3D Ising model discussed above, we can conclude that there is good evidence that for three dimensional systems it is possible to have a strong absence of uniformity in the thermodynamic limit of interesting dynamical quantities like the gap in the spectrum of the generator of the dynamics. Such unexpected phenomenon makes quite hard the discussion of the ergodicity properties of the associated Glauber-type dynamics in the infinite lattice when additional useful properties like attractivity are missing and its solution seems to require new and more sophisticated techniques. We expect, in particular, that one should envisage a method to exploit the fact that the boundary conditions for which the gap is not bounded away from zero in the thermodynamic limit should be very unlikely with respect to the infinite volume Gibbs state.

Let us now describe the contents of the paper. The proof of part (i), discussed in Sect. 2, is organized as follows. Using the methods of [MOS] and some apriori bounds on the moments of the random variable $\varphi(x)$ proved in [CM], one easily improves part (i) of Theorem [CM], by showing that a stronger form of weak dependence on the boundary conditions (strong mixing in the language of [MO1, $\mathrm{MO} 2$, MOS]) holds. Once strong mixing is established, a lower bound of the gap in any large enough cube, uniformly in the volume and in the boundary conditions, follows from the "block dynamics approach" envisaged in [MO1]. This method requires, however, as an input, a lower bound on the gap in a given fixed block. Such a bound would be trivial were the random variables $\varphi(x)$ bounded. In our case the problem requires some extra work and our solution is based upon the so-called "Cheeger inequality" (see [LS]).

The proof of the upper bound of part (ii) is discussed in Sect. 3 and it uses the variational characterization of the gap. More precisely, using a suitable test function $f$, we show the existence of a bottleneck: the system, in order to relax to equilibrium, has to make an excursion to a region of the configuration space of very small equilibrium measure. Finally Sect. 4 contains the proof of the lower bound; our method relies upon a novel recursive estimate of the gap that allows us to overcome the problem of the unboundedness of the variables $\varphi(x)$. 


\section{Preliminaries}

For the reader's convenience we recall most of the basic definitions that were given in $[\mathrm{CM}]$.

1.1. General definitions. We consider the two dimensional lattice $\mathbb{Z}^{2}$ whose elements are called sites and its dual $\mathbb{Z}_{*}^{2}=\mathbb{Z}^{2}+(1 / 2,1 / 2)$. For $x, y \in \mathbb{R}^{2}$ we define two distances

$$
d(x, y)=|x-y|=\sum_{i=1}^{2}\left|x_{i}-y_{i}\right| \quad \text { and } \quad d_{\infty}(x, y)=|x-y|_{\infty}=\max _{i=1,2}\left|x_{i}-y_{i}\right| .
$$

$[x, y]$ is the closed segment with $x, y$ as its endpoints. The edges of $\mathbb{Z}^{2}\left(\mathbb{Z}_{*}^{2}\right)$ are those $e=[x, y]$ with $x, y$ nearest neighbors in $\mathbb{Z}^{2}\left(\mathbb{Z}_{*}^{2}\right)$. Given $e$, the edge of $\mathbb{Z}^{2}$, $e^{*}$ is the unique edge in $\mathbb{Z}_{*}^{2}$ that intersects $e$. The boundary of an edge $e=[x, y]$ is $\delta e=\{x, y\}$. The boundary of a subset of edges $\alpha$ is the set of sites $\delta \alpha$ that belong to an odd number of edges of $\alpha$. A set of edges is called closed if its boundary is empty.

We will often consider our model on a square

$$
Q_{N}=\left\{\begin{array}{ll}
\left\{\left(x_{1}, x_{2}\right) \in \mathbb{Z}^{2}:-L \leqq x_{i} \leqq L, i=1,2\right\} & \text { if } N=2 L+1 \\
\left\{\left(x_{1}, x_{2}\right) \in \mathbb{Z}^{2}:-L+1 \leqq x_{i} \leqq L, i=1,2\right\} & \text { if } N=2 L
\end{array} .\right.
$$

$\Lambda$ and $V$ will denote arbitrary subsets of $\mathbb{Z}^{2}$. If $\Lambda$ is finite we write $\Lambda \subset \subset \mathbb{Z}^{2}$. The cardinality of $\Lambda$ is denoted by $|\Lambda|$. We define four kinds of boundaries:

$$
\begin{aligned}
\partial \Lambda & =\left\{x \in \Lambda: d\left(x, \Lambda^{c}\right)=1\right\}, \\
\bar{\partial} \Lambda & =\left\{x \in \Lambda: d_{\infty}\left(x, \Lambda^{c}\right)=1\right\}, \\
\partial^{+} \Lambda & =\left\{x \in \Lambda^{c}: d(x, \Lambda)=1\right\}, \\
\delta \Lambda & =\left\{e^{*}=[x, y]^{*}:\{x, y\} \cap \Lambda \neq \emptyset,\{x, y\} \cap \Lambda^{c} \neq \emptyset\right\},
\end{aligned}
$$

where $\Lambda^{c}=\mathbb{Z}^{2} \backslash \Lambda$.

$\left(x_{1}, \ldots, x_{n}\right)$ is called a path from $x_{1}$ to $x_{n}$ if $\left|x_{i+1}-x_{i}\right|=1$ for $i=1, \ldots, n-1$. A $*$-path is the same as a path with $\left|x_{i+1}-x_{i}\right|=1$ replaced by $d_{\infty}\left(x_{i}, x_{i+1}\right)=1$. A (*-) path is called self-avoiding if $x_{i} \neq x_{j}$ for all $\{i, j\}$ such that $i \neq j$ and $\{i, j\} \neq\{1, n\}$. If $x_{1}=x_{n}$ the $(*-)$ path is called closed.

$\Lambda \subset \mathbb{Z}^{2}$ is said to be connected (*-connected) if for all $x, y$ in $\Lambda$ there exists a path (*-path) from $x$ to $y$ which is entirely contained in $\Lambda . \Lambda \subset \subset \mathbb{Z}^{2}$ is called simply connected if $\Lambda^{c}$ is $*$-connected. A set of edges $\alpha$ is connected if the union of all its edges is connected in $\mathbb{R}^{2}$.

We denote by $C_{B}$ the set of all finite closed connected set of edges of $\mathbb{Z}_{*}^{2}$. If $\alpha \in C_{B}$ then we define the interior of $\alpha$ (see Fig. 1) as the set of all sites $x=\left(x_{1}, x_{2}\right) \in \mathbb{Z}^{2}$ such that the half line

$$
\left\{x_{1}\right\} \times\left(x_{2},+\infty\right)
$$

intersects $\alpha$ in an odd number of points. The interior of $\alpha$ is denoted by $\bar{\alpha}$ and is always a (possibly disconnected) simply connected subset of $\mathbb{Z}^{2}$ for each $\alpha \in C_{B}$. $C_{B}(V)$ is the set of all $\alpha$ in $C_{B}$ such that $\bar{\alpha} \subset V$. 
$\square \Lambda_{2} \quad \mathrm{~A}_{2}^{\prime \prime} \mathrm{A}_{2}^{\prime}$

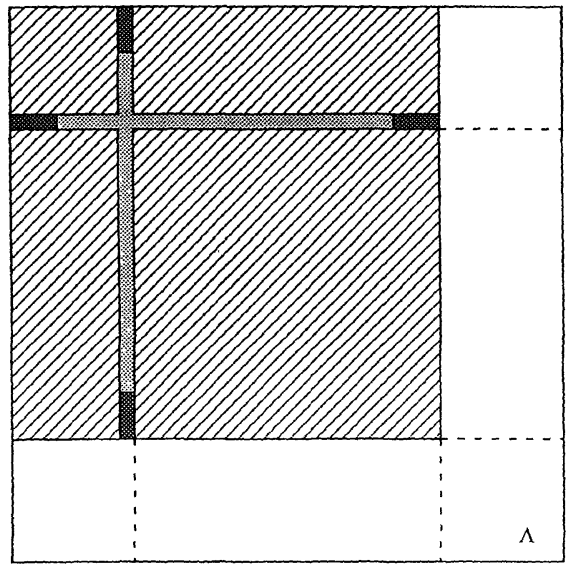

Fig. 1. Proof of Proposition 4.1.

1.2. The SOS model. The configuration space of the model is $\Omega=\mathbb{Z}_{+}^{\mathbb{Z}^{2}}$, or $\Omega_{V}=\mathbb{Z}_{+}^{V}$ for some $V \subset \mathbb{Z}^{2}$. An element of $\Omega_{V}$ will usually be denoted by $\varphi=\{\varphi(x), x \in V\}$. If $U \subset V \subset \mathbb{Z}^{2}$, and $\varphi \in \Omega_{V}$ we denote by $\varphi_{U}$ the restriction of $\varphi$ to the set $U$.

Given $V \subset \subset \mathbb{Z}^{2}$ and some boundary condition (b.c.) $\psi \in \Omega$, one defines the hamiltonian as

$$
\begin{aligned}
H_{V}^{J, h, \psi}(\varphi)= & \frac{1}{2} \sum_{\substack{x, y \in V \\
|x-y|=1}} J(x, y)|\varphi(x)-\varphi(y)| \\
& +\sum_{\substack{x \in V, y \in V c \\
|x-y|=1}} J(x, y)|\varphi(x)-\psi(y)|+h \sum_{x \in V} \varphi(x),
\end{aligned}
$$

and we always assume $0 \leqq J(x, y) \leqq 1$ for all $x, y$. We write $J \in \delta V$ if $J(x, y)<1$ only for the boundary terms, i.e. if $J(x, y)=1$ unless $[x, y]^{*} \in \delta V$. If we take $J(x, y)=0$ for all boundary terms, then we have free boundary conditions that we also denote by

$$
H_{V}^{h, \emptyset}(\varphi)=\frac{1}{2} \sum_{\substack{x, y \in V \\|x-y|=1}}|\varphi(x)-\varphi(y)|+h \sum_{x \in V} \varphi(x) .
$$

The partition function is given by

$$
Z^{J, h, \psi}(V)=\sum_{\varphi \in \Omega_{V}} e^{-\beta H_{V}^{J, h, \psi}(\varphi)} .
$$

Given any set $\alpha$ of dual edges (for instance $\alpha=\delta V$ ) we define

$$
\mid \alpha\}_{J}=\sum_{e^{*}=[x, y]^{*} \in \alpha} J(x, y)
$$

(if $J=1$ everywhere this is just the ordinary length of $\alpha$ ). 
As in [CM] we often assume that $J$ satisfies the hypothesis $H(\Lambda, t)$ for some $\Lambda \subset \subset \mathbb{Z}^{2}$ and $t \geqq 0$, namely that

(i) $J \in \delta \Lambda$,

(ii) $|\alpha|_{J} \geqq t|\alpha|$ for all $\alpha \in C_{B}(\Lambda)$.

We also define

$$
\Delta(J)=\left\{x \in \mathbb{Z}^{2}: J(x, y) \neq 1 \text { for some } y \in \mathbb{Z}^{2}\right\} .
$$

If $H(\Lambda, t)$ holds for $J$ then, clearly, for each $V \subset \Lambda$,

$$
|\Delta(J) \cap V| \leqq|\partial V| \leqq|\delta V|
$$

When $J(x, y)=1$ for all $x, y$, we drop the superscript $J$.

For $U \subset \mathbb{Z}^{2}$, let $\mathbf{F}_{U}$ be the $\sigma$-algebra generated by the collection of sets

$$
\{\varphi \in \Omega: \varphi(x)=n\}_{x \in U, n \in \mathbb{Z}_{+}}
$$

and let $\mathbf{F}=\mathbf{F}_{\mathbb{Z}^{2}}$. The (finite volume) conditional Gibbs measure on $(\Omega, \mathbf{F})$ associated with the Hamiltonian (1.1) is defined as

$$
\mu_{V}^{J, h, \psi}(\varphi)= \begin{cases}\left(Z^{J, h, \psi}(V)\right)^{-1} e^{-\beta H_{V}^{J, h, \psi}(\varphi)} & \text { if } \varphi(x)=\psi(x) \text { for all } x \in V^{c} \\ 0 & \text { otherwise }\end{cases}
$$

We also regard $\mu_{V}^{J, h, \psi}$ as a measure on $\Omega_{V}$ by extending each configuration $\varphi \in \Omega_{V}$ to the whole space in such a way that it agrees with the boundary conditions outside $V$. The expectation with respect to the measure (1.6) is denoted by $\mathbf{E}_{V}^{J, h, \psi}(\cdot)$. The set of probability measures (1.6) satisfies the compatibility conditions

$$
\mu_{\Lambda}^{J, h, \psi}(\varphi)=\sum_{\varphi^{\prime} \in \Omega} \mu_{\Lambda}^{J, h, \psi}\left(\varphi^{\prime}\right) \mu_{V}^{J, h, \varphi^{\prime}}(\varphi) \text { for all } V \subset \Lambda \subset \subset \mathbb{Z}^{2}
$$

We refer to Sect. 1.2 of [CM] for the definition of (infinite volume) Gibbs measure, the definition of increasing (decreasing) functions, and the statement of the FKG properties.

1.3. The dynamics and our results. In the rest of this section, for readability purposes, we assume to have chosen $\beta, h$ and $J$, so we don't write them explicitly if no confusion arises.

The stochastic dynamics we want to study is defined by the Markov generator

$$
\left(L_{V}^{\psi} f\right)(\varphi)=\sum_{x \in V, s= \pm 1} c(x, \varphi, s)\left[f\left(\varphi^{x, s}\right)-f(\varphi)\right]
$$

acting on $L^{2}\left(\Omega, d \mu_{V}^{\psi}\right)$, where

$$
\varphi^{x, s}(y)= \begin{cases}\varphi(y) & \text { if } y \neq x \\ \varphi(y)+s & \text { if } y=x\end{cases}
$$

In (1.8) $\varphi$ denotes a configuration on the whole lattice $\mathbb{Z}^{2}$ which, in view of (1.6), agrees with the b.c. $\psi$ on $V^{c}$. In general we identify $L^{2}\left(\Omega, d \mu_{V}^{\psi}\right)$ with $L^{2}\left(\Omega_{V}, d \mu_{V}^{\psi}\right)$. 
The nonnegative real quantities

$$
c(x, \varphi, s) \quad x \in \mathbb{Z}^{2}, \quad \varphi \in \Omega, s= \pm 1
$$

are the transition rates for the process. We set $c(x, \varphi, s)=0$ if $\varphi^{x, s} \notin \Omega$, that is if $\varphi(x)=1$ and $s=-1$. The assumptions on the transition rates are

$\left(\mathrm{H}_{1}\right)$ Tranlation invariance. If there exists $y$ such that $\tilde{\varphi}(x)=\varphi(x+y)$ for all $x \in \mathbb{Z}^{2}$, then $c(x+y, \varphi, s)=c(x, \tilde{\varphi}, s)$ for all $x \in \mathbb{Z}^{2}, s= \pm 1$.

$\left(\mathrm{H}_{2}\right)$ Nearest neighbor interactions. If $\varphi(y)=\tilde{\varphi}(y)$ for all $y$ such that $d(x, y) \leqq 1$, then $c(x, \varphi, s)=c(x, \tilde{\varphi}, s)$.

$\left(\mathrm{H}_{3}\right)$ Attractivity. If $\varphi \leqq \tilde{\varphi}$ and $\varphi(x)=\tilde{\varphi}(x)$, then

$$
\begin{aligned}
& c(x, \varphi,+) \leqq c(x, \tilde{\varphi},+), \\
& c(x, \varphi,-) \geqq c(x, \tilde{\varphi},-) .
\end{aligned}
$$

$\left(\mathrm{H}_{4}\right)$ Detailed balance. For all $x, \varphi, s$ such that $\varphi^{x, s} \in \Omega$,

$$
\exp \left[-\beta H_{\{x\}}^{\varphi}(\varphi(x))\right] c(x, \varphi, s)=\exp \left[-\beta H_{\{x\}}^{\varphi}(\varphi(x)+s)\right] c\left(x, \varphi^{x, s},-s\right) .
$$

$\left(\mathrm{H}_{5}\right)$ Positivity and boundedness. There exist $c_{m}(\beta, h)>0$ and $c_{M}(\beta, h)<\infty$ such that,

$$
\begin{aligned}
& \inf _{\varphi \in \Omega,} c(x, \varphi, s) \geqq c_{m}(\beta, h), \\
& \sup _{\varphi \in \Omega} \sum_{s= \pm 1} c(x, \varphi, s) \leqq \varphi_{M}(\beta, h)<\infty .
\end{aligned}
$$

Two cases one may want to keep in mind are ( $\chi$ is the characteristic function)

$$
c(x, \varphi, s)=\min \left\{e^{-\beta \Delta_{x, s} H(\varphi)}, 1\right\} \chi\left\{\varphi^{x, s} \in \Omega\right\}
$$

and

$$
c(x, \varphi, s)=\left[1+\exp \left(\beta \Delta_{x, s} H(\varphi)\right)\right]^{-1} \chi\left\{\varphi^{x, s} \in \Omega\right\},
$$

where

$$
\Delta_{x, s} H(\varphi)=H_{\{x\}}^{\varphi}(\varphi(x)+s)-H_{\{x\}}^{\varphi}(\varphi(x)) .
$$

$\left(\mathrm{H}_{1}\right)-\left(\mathrm{H}_{5}\right)$ guarantee that there exists a unique Markov process with semigroup $T_{V}^{\psi}(t)$ and generator $L_{V}^{\psi} . L_{V}^{\psi}$ is a bounded operator on $L^{2}\left(\Omega, d \mu_{V}^{\psi}\right)$. The process has a unique invariant measure given by $\mu_{V}^{\psi}$. Moreover $\mu_{V}^{\psi}$ is reversible with respect to the process, i.e. $L_{V}^{\psi}$ is self-adjoint on $L^{2}\left(\Omega, d \mu_{V}^{\psi}\right)$. Given $\varphi \in \Omega$ we denote by $\varphi_{t}$ the random configuration at time $t$ evolving according to the process, so that

$$
\mathbb{E}_{V}^{\varphi} f\left(\varphi_{t}\right)=\int f\left(\varphi_{t}\right) d \mathbb{P}_{V}^{\varphi}=\left(T_{V}^{\psi}(t)\right) f(\varphi), \quad \forall \varphi \in \Omega \text { such that } \varphi_{V^{c}}=\psi_{V^{c}}
$$

$\mathbb{E}^{\varphi}$ and $\mathbb{P}^{\varphi}$ stand respectively for the expectation and the probability measure associated with the process starting from $\varphi_{V}$ at time zero and subject to b.c. $\varphi_{V^{c}}$.

The attractivity assumption implies (see for instance [L]) $t \geqq 0$.

(1) If $f$ is an increasing function on $\Omega_{V}$ then $T_{V}^{\psi}(t) f$ is also increasing for all

(2) If $\rho_{1}, \rho_{2}$ are two probability measures on $\left(\Omega_{V}, \mathbf{F}_{V}\right)$ such that $\rho_{1} \leqq \rho_{2}$, then $\rho_{1} T_{V}^{\psi}(t) \leqq \rho_{2} T_{V}^{\psi}(t)$ for all $t \geqq 0$. 
(3) For any $\varphi, \varphi^{\prime}$ in $\Omega$ such that $\varphi \leqq \varphi^{\prime}$, the standard coupling $[\mathrm{L}] \mathbb{P}_{V}^{\varphi, \varphi^{\prime}}$ of $\varphi_{t}, \varphi_{t}^{\prime}$ is such that $\mathbb{P}_{V}^{\varphi, \varphi^{\prime}}\left\{\varphi_{t} \leqq \varphi_{t}^{\prime}\right\}=1$, for all $t \geqq 0$.

This last property allows us to define a standard coupling of two Gibbs measures which preserve the order of the b.c. Take in fact $v_{V}^{\psi, \psi^{\prime}}$ as the unique invariant measure of the (standard) coupled process $\left(\varphi_{t}, \varphi_{t}^{\prime}\right)$. Then we have

(1) $v_{V}^{\psi, \psi^{\prime}}\left\{\left(\varphi, \varphi^{\prime}\right): \varphi=\varphi_{0}\right\}=\mu_{V}^{\psi}\left(\varphi_{0}\right)$ for all $\varphi_{0} \in \Omega_{V}$.

(2) $v_{V}^{\psi, \psi^{\prime}}\left\{\left(\varphi, \varphi^{\prime}\right): \varphi^{\prime}=\varphi_{0}\right\}=\mu_{V}^{\psi^{\prime}}\left(\varphi_{0}\right)$ for all $\varphi_{0} \in \Omega_{V}$.

(3) If $\psi \leqq \psi^{\prime}$, then $v_{V}^{\psi, \psi^{\prime}}\left\{\left(\varphi, \varphi^{\prime}\right): \varphi \leqq \varphi^{\prime}\right\}=1$.

A fundamental quantity associated with the dynamics of a reversible system is the gap of the generator, i.e.

$$
\operatorname{gap}^{\psi}(V)=\operatorname{gap}\left(L_{V}^{\psi}\right)=\inf \operatorname{spec}\left(-L_{V}^{\psi} \uparrow \mathbb{1}^{\perp}\right),
$$

where $\mathbb{1}^{\perp}$ is the subspace of $L^{2}\left(\Omega, d \mu_{V}^{\psi}\right)$ orthogonal to the constant functions. The gap can be also characterized as

$$
\operatorname{gap}^{\psi}(V)=\inf _{f \in L^{2}\left(\Omega, d \mu_{V}^{\psi}\right)} \frac{\mathscr{E}_{V}^{\psi}(f, f)}{\operatorname{Var}_{V}^{\psi}(f)},
$$

where $\mathscr{E}$ is the Dirichlet form associated with the generator $L$,

$$
\mathscr{E}_{V}^{\psi}(f, f)=\frac{1}{2} \sum_{\varphi \in \Omega} \sum_{x \in V, s= \pm 1} \mu_{V}^{\psi}(\varphi) c(x, \varphi, s)\left[f\left(\varphi^{x, s}\right)-f(\varphi)\right]^{2}
$$

and $\operatorname{Var}_{V}^{h, \psi}$ is the variance relative to the probability measure $\mu_{V}^{h, \psi}$. Then main result in this paper is

Theorem 1.1. In the same setting as in Theorem CM of the Introduction, if the transition rates satisfy $\left(\mathrm{H}_{1}\right)-\left(\mathrm{H}_{5}\right)$, then we have for all $k=1, \ldots, k_{\max }$,

(i) if $h_{k}^{*}(\beta)<h<h_{k-1}^{*}(\beta)$ then there exist $L_{0}(\beta, h), \kappa(\beta, h)>0$ such that

$$
\inf _{L \geqq L_{0}} \inf _{\psi \in \Omega} \operatorname{gap}^{h, \psi}\left(Q_{L}\right) \geqq \kappa(\beta, h) .
$$

(ii) If $h=h_{k}^{*}(\beta)$ there exists a positive constant $C_{1}(\beta, h)$ such that for all $N>10 / h$,

$$
C_{1}(\beta, h) e^{-100 \beta k N} \leqq \operatorname{gap}^{h, \emptyset}\left(Q_{N}\right) \leqq c_{M}(\beta, h) e^{-\frac{1}{40} \beta N},
$$

where $c_{M}$ is given by (1.11).

\section{Lower Bound on the Gap for $h \neq h_{k}^{*}(\beta)$}

In this section we will show that, if $h_{k}^{*}(\beta)<h<h_{k-1}^{*}(\beta)$, then the finite volume Gibbs measures show a weak dependence (in a strong sense) on the boundary conditions.

As a consequence, we obtain a lower bound for the gap of the dynamics in a finite, large enough square $Q_{N}$. This lower bound is independent of the boundary conditions and of $N$. Thus we get part (i) of Theorem 1.1. 
We will adapt to our model the ideas and techniques developed in [MO1, MOS], supplemented by results of Sect. 3 of [CM] and by Proposition 7.1 of [CM]. As in [CM] we often need a small constant, so we set

$$
\zeta=1000^{-1} \text {. }
$$

2.1. Strong mixing. Following the basic result of [MOS], we strengthen the result of Proposition 7.1 of [CM].

Theorem 2.1 (Strong Mixing). Let $\beta$ be large enough and $h \in\left(h_{k}^{*}(\beta), h_{k-1}^{*}(\beta)\right)$, with $1 \leqq k \leqq k_{\max }=\left\lfloor e^{\frac{\beta \zeta}{20}}\right\rfloor$. Then there exists $L_{0}(\beta, h), C(\beta, h)$ and $m(\beta, h)>0$ such that, for any $L \geqq L_{0}$, any $\Delta \subset Q_{L}$ and any $y \in \partial^{+} Q_{L}$, we have

$$
\sup _{\substack{\psi, \psi^{\prime} \in \Omega \\ \psi^{\prime}(x)=\psi(x), \forall x \neq y}} \sup _{A \in \mathbf{F}_{\Delta}}\left|\mu_{Q_{L}}^{h, \psi}(A)-\mu_{Q_{L}}^{h, \psi^{\prime}}(A)\right| \leqq C \sum_{x \in \Delta} e^{-m d(x, y)} .
$$

Remark. Following [MO1], we will say that $\operatorname{SM}(L, C, m)$ holds if the above inequality with the prescribed constants is valid for a square $Q_{L}$.

Proof. If the random variables were bounded then the result would simply follow from Proposition 7.1 of [CM], thanks to the main theorem of [MOS] which states that, for discrete bounded spin systems with finite range interaction, weak mixing implies strong mixing in two dimensions.

Thus we have to take care of the unboundedness of the $\varphi(x)$ 's. If one examines closely the arguments of [MOS] one finds out that the two basic ingredients (besides the dimensionality and the finite range condition) are the following:

(i) Weak mixing in the form stated in Proposition 7.1 of [CM].

(ii) Let $C, m$ be the "weak mixing" constants appearing in Proposition 7.1 of [CM], and let $l_{0}(\beta, h)$ be such that

$$
C \sum_{j=l_{0}}^{\infty} e^{-m j} \leqq \frac{1}{2}
$$

Then there exists $\bar{N}(\beta, h) \geqq l_{0}$ such that

$$
\inf _{\psi \in \Omega} \mu_{Q_{\bar{N}}}^{\psi}\left\{\varphi(x)=\bar{\varphi}(x) \forall x \in Q_{\bar{N}}\right\}>0 \quad \text { for some } \bar{\varphi} \in \Omega \text {. }
$$

While for bounded spins with finite range interaction, (2.1) is a trivial consequence of the boundedness of the interaction, in our case (2.1) follows from part (ii) of Proposition 3.2 of $[\mathrm{CM}]$, if one chooses the reference configuration $\bar{\varphi}$ identically equal to +1 , and $\bar{N}=l_{0} \vee\lfloor 8 / h+1\rfloor$. In fact from the FKG inequality one gets

$$
\inf _{\psi \in \Omega} \mu_{Q_{\bar{N}}}^{\psi}\left\{\varphi(x)=+1 \forall x \in Q_{\bar{N}}\right\} \geqq \prod_{x \in Q_{\bar{N}}} \inf _{\psi \in \Omega} \mu_{Q_{\bar{N}}}^{\psi}\{\varphi(x)=+1\} \geqq b_{2}(\beta, h)^{\left|Q_{\bar{N}}\right|} .
$$

Thus (i) and (ii) hold also in our case and the theorem follows.

2.2. Proof of part (i) of Theorem 1.1. We are finally in a position to prove a lower bound for the gap of the dynamics defined in Sect. 1.3 in any finite, large enough square $Q_{N}$, independent of the boundary condition and of $N$. 
We follow very closely Sect. 4 of [MO1]. Given an even integer $L_{0}=2 K_{0}$ and a cube $Q_{N}, N=n L_{0}$, we consider its covering by squares $Q_{L_{0}}^{i}$ such that if two different squares $Q_{L_{0}}^{i}$ and $Q_{L_{0}}^{j}$ overlap then necessarily each one of them is translated by $K_{0}$ of the other along at least one of the two coordinate axes. Let

$$
\mathscr{D}_{n}=\left\{Q_{L_{0}}^{i}\right\}
$$

and consider the block-dynamics defined in the Appendix, determined by $\beta, h$, the b.c. $\psi$ and the collection $\mathscr{D}_{n}$, through the Markov generator $L_{\mathscr{D}}^{h, \psi}$ given by (A1.1). The same proof of Theorem 4.1 of [MO1] shows that, if $\operatorname{SM}\left(L_{0}, C, m\right)$ holds and moreover $L_{0}$ is so large that

$$
C L_{0}^{2} \exp \left(-m \sqrt{L_{0}}\right) \leqq \frac{1}{L_{0}^{4}},
$$

then the gap of $L_{\mathscr{D}_{n}}^{h, \psi}$ is bounded away from zero uniformly in $\psi$ and in $N=n L_{0}$. Thus if we choose $L_{0}=L_{0}(\beta, h)$ large enough so that both Theorem 2.1 and (2.2) are satisfied we get

$$
\inf _{\psi \in \Omega} \inf _{n \geqq 1} \operatorname{gap}\left(L_{\mathscr{D}_{n}}^{h, \psi}\right) \geqq \kappa_{1}(\beta, h)>0 .
$$

In Proposition 2.2, on the other side, we will show that, considering the usual single site dynamics on each block $Q_{L_{0}}^{i}$, if $L_{0}>\lfloor 8 / h+1\rfloor$, we have

$$
\inf _{\psi \in \Omega} \operatorname{gap}\left(L_{Q_{L_{0}}}^{h, \psi}\right) \geqq \kappa_{2}(\beta, h)>0 .
$$

Finally, Proposition A1.1 gives an estimate for the gap of the single site dynamics in the full volume $Q_{N}$, in terms of the quantities appearing in (2.3), (2.4). Thus, assuming to have chosen $L_{0}$ such that $L_{0} \geqq\lfloor 8 / h+1\rfloor$ also holds, we get

$$
\inf _{N=n L_{0}, n \in \mathbb{Z}_{+}} \inf _{\psi \in \Omega} \operatorname{gap}\left(L_{Q_{N}}^{h, \psi}\right) \geqq \frac{1}{4} \kappa_{1} \kappa_{2}>0 .
$$

The factor $\frac{1}{4}$ is the "overlapping" factor appearing as the last term in (A1.2).

The extension of the theorem to all $N \geqq L_{0}$ is straightforward after one realizes that Theorem 4.1 of [MO1] is valid for any coverings $\left\{Q_{L_{0}}^{i}\right\}$ of $Q_{N}$ such that the following holds: for each site $x \in Q_{N}$ there is a square $Q_{L_{0}}^{i}$,

$$
d\left(x,\left(Q_{L_{0}}^{i}\right)^{c} \backslash\left(Q_{N}\right)^{c}\right) \geqq \frac{1}{10} L_{0} .
$$

In this way we can "cover" any square $Q_{N}$ with $N$ greater than say $10 L_{0}$.

We are thus left with the proof of (2.4).

Proposition 2.2. For each $\beta, h>0, N \geqq N_{1}(h)=\lfloor 8 / h+1\rfloor$, we have (remember that we always assume $0 \leqq J(x, y) \leqq 1)$

$$
g_{N}(\beta, h) \equiv \inf _{J \in \delta Q_{N}} \inf _{\psi \in \Omega} \operatorname{gap}\left(L_{Q_{N}}^{J, h, \psi}\right)>0
$$

Proof. We recall that $J \in \delta Q_{N}$ means (see Sect. 1.2) that $J=1$ everywhere with the possible exception of the boundary terms. Let then $\Lambda=Q_{N}$. As we did in Sect. 3 
of $[\mathrm{CM}]$ we define

$$
\bar{H}_{V}^{h, \infty}(\varphi)=\frac{1}{2} \sum_{\substack{x, y \in V \\|x-y|=1}}|\varphi(x)-\varphi(y)|-\sum_{\substack{x \in V, y \in V^{c} \\|x-y|=1}} \varphi(x)+h \sum_{x \in V} \varphi(x),
$$

which satisfies

$$
(n-1)|\delta V|_{J}+H_{V}^{J, h, 1}(\varphi) \geqq H_{V}^{J, h, n}(\varphi) \geqq(n-1)|\delta V|_{J}+\bar{H}_{V}^{h, \infty}(\varphi) .
$$

For $X \subset \Omega_{\Lambda}$ we define $\delta X$ as the set of all $\varphi \in X$ such that there exists $\varphi^{\prime} \in X^{c}$ with

$$
\sum_{x \in \Lambda}\left|\varphi(x)-\varphi^{\prime}(x)\right|=1
$$

The proof is based on the so-called Cheeger inequality given in [LS], which in our case says

$$
\operatorname{gap}\left(L_{\Lambda}^{J, h, \psi}\right) \geqq \frac{q^{2}}{8 M}
$$

where, letting $\mu=\mu_{\Lambda}^{J, h, \psi}$,

$$
q=\inf _{\substack{X \subset \Omega_{\Lambda} \\ 0<\mu(X) \leqq \frac{1}{2}}} \frac{1}{\mu(X) \mu\left(X^{c}\right)} \sum_{\varphi \in X} \mu(\varphi) \sum_{(x, s): \varphi^{x, s} \in X^{c}} c(x, \varphi, s) \geqq \inf _{\substack{X \subset \Omega_{\Lambda} \\ 0<\mu(X) \leqq \frac{1}{2}}} c_{m}(\beta, h) \frac{\mu(\partial X)}{\mu(X)},
$$

and

$$
M=\sup _{\varphi \in \Omega_{\Lambda}} \sum_{x \in \Lambda} \sum_{s= \pm 1} c(x, \varphi, s) \leqq|\Lambda| c_{M}(\beta, h) .
$$

Thanks to the boundedness of the transition rates (1.11), the proposition is thus proven if we can show that

$$
\inf _{J \in \delta V} \inf _{\psi \in \Omega} \inf _{\substack{X \subset \Omega_{\Lambda} \\ 0<\mu_{\Lambda}^{J, h, \psi}(X) \leqq \frac{1}{2}}} \frac{\mu_{\Lambda}^{J, h, \psi}(\partial X)}{\mu_{\Lambda}^{J, h, \psi}(X)}>0 .
$$

We denote by $\varphi_{1}$ the configuration on $\Lambda$ which is equal to 1 everywhere, and we consider two cases depending on whether $\varphi_{1}$ belongs to $X$ or not.

- $\varphi_{1} \notin X$

For each $\varphi \in X$ we set

$$
\begin{aligned}
& m(\varphi)=\inf \{i: \varphi \wedge i \in X\}, \\
& \varphi^{\prime}=\varphi \wedge m(\varphi) \text { and } \varphi^{\prime \prime}=\varphi \wedge(m(\varphi)-1),
\end{aligned}
$$

so that $\varphi^{\prime} \in X$ and $\varphi^{\prime \prime} \in X^{c}$. If we define $T \varphi=\varphi^{\prime}$, then we claim that

$$
\frac{\mu_{\Lambda}^{J, h, \psi}(X)}{\mu_{\Lambda}^{J, h, \psi}(T X)} \leqq \sum_{\varphi \in \tilde{\Omega}_{\Lambda}} e^{-\beta \bar{H}_{\Lambda}^{h, \infty}(\varphi)} \quad \forall \psi \in \Omega
$$


where $\tilde{\Omega}_{\Lambda}=\{0,1,2, \ldots\}^{\Lambda}$. To prove $(2.8)$ one has to observe that, since $\varphi(x) \geqq \varphi(y)$ implies $\varphi^{\prime}(x) \geqq \varphi^{\prime}(y)$, then

$$
\begin{aligned}
& |\varphi(x)-\varphi(y)|=\left|\varphi^{\prime}(x)-\varphi^{\prime}(y)\right|+\left|\left(\varphi-\varphi^{\prime}\right)(x)-\left(\varphi-\varphi^{\prime}\right)(y)\right| \\
& J(x, y)|\varphi(x)-\psi(y)| \geqq-\left(\varphi-\varphi^{\prime}\right)(x)+J(x, y)\left|\varphi^{\prime}(x)-\psi(y)\right| .
\end{aligned}
$$

This implies

$$
H_{\Lambda}^{J, h, \psi}(\varphi) \geqq H_{\Lambda}^{J, h, \psi}\left(\varphi^{\prime}\right)+\bar{H}_{\Lambda}^{h, \infty}\left(\varphi-\varphi^{\prime}\right) .
$$

So, if we let $Z=Z^{J, h, \psi}(\Lambda)$, we have

$$
\begin{aligned}
\mu_{\Lambda}^{J, h, \psi}(X) & =Z^{-1} \sum_{\varphi \in \Omega_{\Lambda}} e^{-\beta H_{\Lambda}^{J, h, \psi}(\varphi)}=Z^{-1} \sum_{\tilde{\varphi} \in T X} \sum_{\varphi \in \Omega_{\Lambda}: T \varphi=\tilde{\varphi}} e^{-\beta H_{\Lambda}^{J, h, \psi}(\varphi)} \\
& \leqq Z^{-1} \sum_{\tilde{\varphi} \in T X} e^{-\beta H_{\Lambda}^{J, h, \psi}(\tilde{\varphi})} \sum_{\varphi \in \Omega_{\Lambda}: T \varphi=\tilde{\varphi}} e^{-\beta \bar{H}_{\Lambda}^{h, \infty}(\varphi-\tilde{\varphi})} \\
& \leqq \mu_{\Lambda}^{J, h, \psi}(T X) \sum_{\varphi \in \tilde{\Omega}_{\Lambda}} e^{-\beta \bar{H}_{\Lambda}^{h, \infty}(\varphi)}
\end{aligned}
$$

To complete the proof we are going to use the following facts: $\tilde{\varphi}$, and

(a) For each $X$, for each $\tilde{\varphi} \in T X$, there exists $\bar{\varphi} \in \partial X$ such that $\tilde{\varphi}-1 \leqq \bar{\varphi} \leqq$

(b) $\left|H_{\Lambda}^{J, h, \psi}(\tilde{\varphi})-H_{\Lambda}^{J, h, \psi}(\bar{\varphi})\right| \leqq 5 N^{2}$.

Statement (a) is a trivial consequence of the definition of $T \varphi$, while (b) follows easily from (a). Given (a) and (b), we can proceed as follows:

$$
\begin{aligned}
\mu_{\Lambda}^{J, h, \psi}(T X) & \leqq Z^{-1} e^{5 \beta N^{2}} \sum_{\tilde{\varphi} \in T X} e^{-\beta H_{\Lambda}^{J, h, \psi}(\bar{\varphi})} \\
& =Z^{-1} e^{5 \beta N^{2}} \sum_{\bar{\varphi} \in \partial X} \#\{\tilde{\varphi} \in T X: \tilde{\varphi} \rightarrow \bar{\varphi}\} e^{-\beta H_{\Lambda}^{J, h, \psi}(\bar{\varphi})} \\
& \leqq e^{5 \beta N^{2}} 2^{N^{2}} \mu_{\Lambda}^{J, h, \psi}(\partial X)
\end{aligned}
$$

The proof of (2.7) when $\varphi_{1} \notin X$, follows then from (2.10), (2.11) and Proposition 3.1 of [CM] (the fact that $\Omega_{\Lambda}$ should be replaced by $\tilde{\Omega}_{\Lambda}$ is not essential).

- $\varphi_{1} \in X$.

The key ingredient is the following observation

(c) there exists $s(\beta, h, N)$ such that for all $X \in \Omega_{\Lambda}$ with $\mu_{\Lambda}^{J, h, \psi}(X) \leqq 1 / 2$ for some $\psi \in \Omega$, there exists $\varphi \in X^{c}$ with $\sup _{x} \varphi(x) \leqq s(\beta, h, N)$.

Proof of $(c)$. By Proposition 3.2 of $[\mathrm{CM}]$ and Chebyshev inequality, we have

$$
\mu_{\Lambda}^{J, h, \psi}\left\{\sup _{x} \varphi(x)>r\right\} \leqq \sum_{x \in \Lambda} \mu_{\Lambda}^{J, h, \psi}\{\varphi(x)>r\} \leqq \frac{1}{r}|\Lambda| b_{1}(\beta, h, 1) .
$$


So, by taking $s(\beta, h, N)=\left[4|\Lambda| b_{1}(\beta, h, 1)\right]^{-1}$, we get

$$
\mu_{\Lambda}^{J, h, \psi}\left\{\sup _{x} \varphi(x) \leqq s(\beta, h, N)\right\} \geqq \frac{3}{4},
$$

which implies $(c)$.

Choose now some $\tilde{\varphi} \in X^{c}$ such that $\sup _{x} \tilde{\varphi}(x) \leqq s$. Since $\varphi_{1} \in X$, there exists $\bar{\varphi} \in \partial X$ such that $\varphi_{1} \leqq \bar{\varphi} \leqq \tilde{\varphi}$. Hence, using the trivial bounds

$$
H_{\Lambda}^{J, h, 1}(\varphi) \leqq 5|\Lambda| \sup _{x} \varphi(x)
$$

we obtain (remember (2.6)),

$$
\begin{aligned}
\mu_{\Lambda}^{J, h, \psi}(\partial X) & \geqq \mu_{\Lambda}^{J, h, \psi}(\bar{\varphi})=\left(Z^{J, h, \psi}(\Lambda)\right)^{-1} e^{-\beta H_{\Lambda}^{J, h, \psi}(\bar{\varphi})} \\
& \geqq e^{-\beta H_{\Lambda}^{J, h, 1}(\bar{\varphi})}\left[\sum_{\varphi \in \Omega_{\Lambda}} e^{-\beta \bar{H}_{\Lambda}^{h, \infty}(\varphi)}\right]^{-1} \\
& \geqq\left[\sum_{\varphi \in \Omega_{\Lambda}} e^{-\beta \bar{H}_{\Lambda}^{h, \infty}(\varphi)}\right]^{-1} e^{-5 \beta|\Lambda| s(\beta, h, N)}
\end{aligned}
$$

This completes the proof of (2.7) and Proposition 2.2.

\section{Upper Bound on the Gap at $h_{k}^{*}(\beta)$}

In this section we prove the upper bound in part (ii) of Theorem 1.1. More precisely, we have

Theorem 3.1. Let $\beta$ be large enough and let $k \in \mathbb{Z}_{+}$such that $1 \leqq k \leqq k_{\max }=$ $\left\lfloor e^{\frac{\beta \zeta}{20}}\right\rfloor$. If $N>10 / h_{k}^{*}(\beta)$, then

$$
\operatorname{gap}\left(L_{Q_{N}}^{h_{k}^{*}(\beta), \emptyset}\right) \leqq c_{M}\left(\beta, h_{k}^{*}(\beta)\right) e^{-\frac{1}{40} \beta N},
$$

where $c_{M}(\beta, h)$ is defined in (1.11).

We set for simplicity $h^{*}=h_{k}^{*}(\beta)$ and $\Lambda=Q_{N}$. In order to find an upper bound on $\operatorname{gap}^{h^{*}, \emptyset}(\Lambda)$ we are going to use the "look for the bottleneck" approach, i.e. we take advantage of the variational characterization for the gap (1.12) and choose an appropriate test function $f_{0}$ which illustrates how the system, in order to relax to equilibrium, has to make an excursion to a very unlikely region.

Given $\varphi \in \Omega$ we set

$$
\sigma(x)=\operatorname{sign}(\varphi(x)-k-1 / 2)
$$

and, for $U \subset \subset \mathbb{Z}^{2}$

$$
\begin{aligned}
& M_{U}(\sigma)=M_{U}(\sigma(\varphi))=\sum_{x \in U} \sigma(x) \\
& m_{U}(\sigma)=m_{U}(\sigma(\varphi))=|U|^{-1} M_{U}(\sigma)
\end{aligned}
$$


In analogy with the solution of the similar problem for the $2 \mathrm{D}$ Ising model in the phase coexistence region, we take as a test function

$$
f_{0}(\varphi)=\chi\left\{M_{\Lambda}(\varphi)>0\right\}-\chi\left\{M_{\Lambda}(\varphi)<0\right\} .
$$

Using (1.11), we find

$$
\begin{gathered}
\mathscr{E}_{\Lambda}^{h^{*}, \emptyset}\left(f_{0}, f_{0}\right) \leqq \mu_{\Lambda}^{h^{*}, \emptyset}\left\{\left|M_{\Lambda}(\varphi)\right| \leqq 2\right\} \sup _{\varphi \in \Omega} \sum_{s= \pm} c_{\Lambda}^{h^{*}, \emptyset}(x, \varphi, s) \\
\leqq c_{M}(\beta, h) \mu_{\Lambda}^{h^{*}, \emptyset}\left\{\left|M_{\Lambda}(\varphi)\right| \leqq 2\right\}
\end{gathered}
$$

while

$$
\begin{aligned}
\operatorname{Var}_{\Lambda}^{h^{*}, \emptyset}\left(f_{0}\right)= & \mu_{\Lambda}^{h^{*}, \emptyset}\left\{M_{\Lambda}(\varphi) \neq 0\right\}-\left(\mu_{\Lambda}^{h^{*}, \emptyset}\left\{M_{\Lambda}(\varphi)>0\right\}\right. \\
& \left.-\mu_{\Lambda}^{h^{*}, \emptyset}\left\{M_{\Lambda}(\varphi)<0\right\}\right)^{2} .
\end{aligned}
$$

The proof of Theorem 3.1 is then a consequence of (1.12), (3.1), (3.2) and Lemmas 3.3, 3.4 proven below.

3.1. Bound on the Dirichlet form. We want to show that, if $\beta$ is large enough and $N \geqq 10 / h_{k}^{*}(\beta)$, then

$$
\mu_{\Lambda}^{h^{*}, \emptyset}\left\{\left|M_{\Lambda}(\varphi)\right| \leqq 2\right\} \leqq e^{-\operatorname{cost} \beta|\delta \Lambda|} .
$$

The idea is the following (see also [S2]). Take $\Lambda^{\prime} \subset \Lambda$. Then either there is a "contour" separating the phase $\sigma=1$ from the phase $\sigma=-1$ or there is a circuit surrounding $\Lambda^{\prime}$, where $\sigma$ is either all plus or all minus. So we need to prove that

- the probability of having a large contour of length $l$ with free b.c. goes like $\exp (-\operatorname{cost} \beta l)$

- the probability of having $\left|M_{\Lambda^{\prime}}\right| \leqq 2$ conditioned to the existence of a $(+)$ circuit or a $(-)$ circuit surrounding $\Lambda^{\prime}$ goes like $\exp \left(-\operatorname{cost} \beta\left|\delta \Lambda^{\prime}\right|\right)$.

Before stating the next lemma we have introduce another definition. Given $\sigma \in\{-1,1\}^{V}$, let $\sigma_{+} \in\{-1,1\}^{\mathbb{Z}^{2}}$ be defined by

$$
\sigma_{+}(x)=\left\{\begin{array}{ll}
\sigma(x) & \text { if } x \in V \\
+1 & \text { if } x \in V^{c}
\end{array} .\right.
$$

The set $\left\{e^{*}: e=[x, y], \sigma_{+}(x) \neq \sigma_{+}(y)\right\}$ can be written as a union of its connected components $\alpha_{1}, \ldots, \alpha_{r}$, where $\alpha_{i} \in C_{B}(V)$. A closed set of dual edges $\alpha$ is called a contour for $\sigma$ if $\alpha=\alpha_{i}$, for some $i$.

Lemma 3.2. Let $\beta$ be large enough, $1 \leqq k \leqq k_{\max }, \Lambda=Q_{N}$. For each $\alpha \in C_{B}(\Lambda)$, let $\alpha_{0}=\alpha \backslash \delta \Lambda$. Then, if $\alpha \cap \delta \Lambda \neq \emptyset$ and $\left|\alpha_{0}\right|>N / 100$, we have

$$
\mu_{\Lambda}^{h_{k}^{*}(\beta), \emptyset}\left\{\varphi \in \Omega_{\Lambda}: \alpha \text { is a contour for } \sigma(\varphi)\right\} \leqq e^{-0.99 \beta\left|\alpha_{0}\right|} .
$$

Proof. Let $h^{*}=h_{k}^{*}(\beta)$. Given $\alpha \in C_{B}(\Lambda)$ we define the event

$$
X_{\alpha}=\left\{\varphi \in \Omega_{\Lambda}: \alpha \text { is a contour for } \varphi\right\} .
$$


We also let $\Lambda_{1}=\bar{\alpha}, \Lambda_{2}=\Lambda \backslash \bar{\alpha}$ and

$$
\begin{aligned}
& \Delta_{1}=\left\{x \in \Lambda_{1}: d\left(x, \Lambda_{2}\right)=1\right\}, \\
& \Delta_{2}=\left\{x \in \Lambda_{2}: d\left(x, \Lambda_{1}\right)=1\right\} .
\end{aligned}
$$

We observe that both $\Lambda_{1}$ and $\Lambda_{2}$ are simply connected. In fact $\Lambda_{1}$ is the interior of a closed connected set of dual edges, and $\Lambda_{2}$ is the interior of $(\alpha \cup \delta \Lambda) \backslash(\alpha \cap \delta \Lambda)$ which is again a closed connected set of dual edges because $\alpha$ touches $\delta \Lambda$. So we have

$$
X_{\alpha} \subset X_{\alpha}^{1} \cup X_{\alpha}^{2}
$$

where

$$
\begin{aligned}
& X_{\alpha}^{1}=\left\{\varphi \in \Omega_{\Lambda}: \sigma(x)=-1 \forall x \in \Delta_{1}, \text { and } \sigma(x)=+1 \forall x \in \Delta_{2}\right\}, \\
& X_{\alpha}^{2}=\left\{\varphi \in \Omega_{\Lambda}: \sigma(x)=+1 \forall x \in \Delta_{1}, \text { and } \sigma(x)=-1 \forall x \in \Delta_{2}\right\} .
\end{aligned}
$$

In the following we are going to show how to estimate $\mu_{\Lambda}^{h^{*}, \emptyset}\left(X_{\alpha}^{1}\right) \cdot \mu_{\Lambda}^{h^{*}, \emptyset}\left(X_{\alpha}^{2}\right)$ can be dealt with in the same way. Choose now the couplings $J(e)$ which correspond to free b.c. on the boundary of $\Lambda$, i.e.

$$
J(x, y)=\left\{\begin{array}{ll}
1 & \text { if }[x, y]^{*} \notin \delta \Lambda \\
0 & \text { if }[x, y]^{*} \in \delta \Lambda
\end{array} .\right.
$$

In this way, since, for $\varphi \in X_{\alpha}^{1}$,

$$
H_{\Lambda}^{h^{*}, \emptyset}(\varphi)=H_{\Lambda_{1}}^{J, h^{*}, k}(\varphi)+H_{\Lambda_{2}}^{J, h^{*}, k+1}(\varphi)+\left|\alpha_{0}\right|,
$$

we can write

$$
\mu_{\Lambda}^{h^{*}, \emptyset}\left(X_{\alpha}^{1}\right)=\frac{e^{-\beta\left|\alpha_{0}\right|} Z^{J, h^{*}, k}\left(\Lambda_{1}\right) Z^{J, h^{*}, k+1}\left(\Lambda_{2}\right)}{Z^{h^{*}, \emptyset}(\Lambda)} .
$$

At this point our strategy depends on whether $|\alpha \cap \delta \Lambda|$ is greater than or less than $\frac{1}{2}|\delta \Lambda|$. In the first case we estimate the denominator as

$$
Z^{h^{*}, \emptyset}(\Lambda) \geqq Z^{J, h^{*}, k}\left(\Lambda_{1}\right) Z^{J, h^{*}, k}\left(\Lambda_{2}\right),
$$

while in the second case we write

$$
Z^{h^{*}, \emptyset}(\Lambda) \geqq Z^{J, h^{*}, k+1}\left(\Lambda_{1}\right) Z^{J, h^{*}, k+1}\left(\Lambda_{2}\right) .
$$

We treat only the first case because the second is identical. So, assuming $|\alpha \cap \delta \Lambda| \geqq$ $\frac{1}{2}|\delta \Lambda|$, we get

$$
\mu_{\Lambda}^{h^{*}, \emptyset}\left(X_{\alpha}^{1}\right) \leqq e^{-\beta\left|\alpha_{0}\right|} \frac{Z^{J, h^{*}, k+1}\left(\Lambda_{2}\right)}{Z^{J, h^{*}, k}\left(\Lambda_{2}\right)} .
$$

What is left to complete the proof is to convince ourselves that (remember the definition of $H(\Lambda, t)$ given before (1.5) and that $\zeta=1000^{-1}$ )

$$
H\left(\Lambda_{2}, 10 \zeta\right) \text { holds for } J \text {. }
$$


In fact, assuming $(*)$, we apply Corollary 4.2 of $[\mathrm{CM}]$ to estimate the above quotient and obtain

$$
\mu_{\Lambda}^{h^{*}, \emptyset}\left(X_{\alpha}^{1}\right) \leqq \exp \left[-\beta\left|\alpha_{0}\right|+\left|\delta \Lambda_{2}\right| e^{-\frac{1}{4} \beta \zeta}\right] \leqq e^{-\beta\left|\alpha_{0}\right|+\left|\alpha_{0}\right|+|\delta \Lambda|} \leqq e^{\left|\alpha_{0}\right|(-\beta+401)},
$$

where we have used $\left|\alpha_{0}\right| \geqq N / 100$.

Proof of $(*)$. Let $\eta \in C_{B}\left(\Lambda_{2}\right)$. We are going to show that $|\eta|_{J} \geqq \frac{1}{3}|\eta|$, which is more than enough. We recall that with our choice of $J,|\eta|_{J}=|\eta \backslash \delta \Lambda|$ and that $\delta \Lambda_{2}=\alpha_{0} \cup(\delta \Lambda \backslash \alpha)$, so that

$$
|\eta \cap \delta \Lambda| \leqq\left|\delta \Lambda_{2} \cap \delta \Lambda\right|=|\delta \Lambda \backslash \alpha| \leqq \frac{1}{2}|\delta \Lambda|
$$

because of what we assumed right before (3.5). We consider three cases:

(1) If $\eta$ does not touch any two opposite sides of $\Lambda$, then $|\eta| \geqq 2|\eta \cap \delta \Lambda|$, so $|\eta|_{J}=|\eta|-|\eta \cap \delta \Lambda| \geqq \frac{1}{2}|\eta|$.

(2) If $\eta$ touches exactly 3 sides of $\Lambda$, then, since it is closed, $|\eta \backslash \delta \Lambda| \geqq \frac{1}{4}|\delta \Lambda|$, so, by (3.6), $|\eta \cap \delta \Lambda| \leqq 2|\eta \backslash \delta \Lambda|$ which implies $|\eta|_{J} \geqq \frac{1}{3}|\eta|$.

(3) If $\eta$ touches all 4 sides of $\Lambda$, then $|\eta| \geqq|\delta \Lambda|$, so $|\eta|_{J} \geqq \frac{1}{2}|\eta|$.

This proves $(*)$.

Thus we have obtained

$$
\mu_{\Lambda}^{h^{*}, \emptyset}\left(X_{\alpha}\right) \leqq 2 e^{\left|\alpha_{0}\right|(-\beta+401)} \leqq e^{-0.99 \beta\left|\alpha_{0}\right|} .
$$

We can now state our first key estimate

Lemma 3.3. Let $\beta$ be large enough, $1 \leqq k \leqq k_{\max }$ and $\Lambda=Q_{N}$. Then, if $N \geqq 1000$,

$$
\mu_{\Lambda}^{h_{k}^{*}(\beta), \emptyset}\left\{\varphi \in \Omega_{\Lambda}:\left|M_{\Lambda}(\varphi)\right| \leqq 2\right\} \leqq e^{-\frac{1}{12} \beta N} .
$$

Proof. We borrow the basic idea from [S2]. Let $h^{*}=h_{k}^{*}(\beta)$. Consider a square $\Lambda^{\prime}=Q_{N^{\prime}}$ of side $N^{\prime}=N-2\lfloor N / 20\rfloor$. In this way $d\left(\delta \Lambda, \delta \Lambda^{\prime}\right)=\lfloor N / 20\rfloor$. It is then easy to check that

$$
\mu_{\Lambda}^{h^{*}, \emptyset}\left\{\left|M_{\Lambda}(\varphi)\right| \leqq 2\right\} \leqq \mu_{\Lambda}^{h^{*}, \emptyset}\left\{\left|m_{\Lambda^{\prime}}(\varphi)\right| \leqq \frac{3}{8}\right\} .
$$

Following [S2] we consider the events

$$
Y^{ \pm}=\left\{\varphi \in \Omega_{\Lambda}: \begin{array}{l}
\text { there exists a connected set } R \subset \Lambda \backslash \Lambda^{\prime}, \text { surrounding } \Lambda^{\prime} \\
\text { such that } \sigma(x)= \pm 1 \text { for all } x \in R
\end{array}\right\}
$$

and, letting $Y=Y^{+} \cup Y^{-}$, we write

$$
\begin{aligned}
\mu_{\Lambda}^{h^{*}, \emptyset}\left\{\left|m_{\Lambda^{\prime}}(\varphi)\right| \leqq \frac{3}{8}\right\} \leqq & \mu_{\Lambda}^{h^{*}, \emptyset}\left\{m_{\Lambda^{\prime}}(\varphi) \leqq \frac{3}{8} \mid Y^{+}\right\} \\
& +\mu_{\Lambda}^{h^{*}, \emptyset}\left\{m_{\Lambda^{\prime}}(\varphi) \geqq-\frac{3}{8} \mid Y^{-}\right\}+\mu_{\Lambda}^{h^{*}, \emptyset}\left(Y^{c}\right) .
\end{aligned}
$$


The idea is to show that the first two terms are small because of Corollary 4.3 of $[\mathrm{CM}]$, while the last term is small because of Lemma 3.2. The first two terms can be taken care of in the same way, so we will deal only with the first.

Consider $\Lambda^{\prime}$ as a disjoint union of $N^{\prime}$ horizontal strips $S_{j}$, each strip being a $N^{\prime} \times 1$ rectangle. Accordingly write

$$
\Lambda^{\prime}=S_{1} \cup S_{2} \cup \cdots \cup S_{N^{\prime}} .
$$

By conditioning on the "most external" $R$ which surrounds $\Lambda^{\prime}$, and using the FKG inequality and the Markov property as we did in Proposition A1.1 of [CM], we find

$$
\mu_{\Lambda}^{h^{*}, \emptyset}\left\{m_{\Lambda^{\prime}}(\varphi) \leqq \frac{3}{8} \mid Y^{+}\right\} \leqq \sup _{\substack{V: \Lambda^{\prime} \subset V \subset \Lambda \\ \text { connected and } \\ \text { simply connected }}} \mu_{V}^{h^{*}, k+1}\left\{m_{\Lambda^{\prime}}(\varphi) \leqq \frac{3}{8}\right\}
$$

Applying Corollary 4.3 of $[\mathrm{CM}]$ to each $S_{j}$ we get

$$
\begin{aligned}
\mu_{\Lambda}^{h^{*}, \emptyset}\left\{m_{\Lambda^{\prime}}(\varphi) \leqq \frac{3}{8} \mid Y^{+}\right\} & \leqq \sup _{\substack{V: \Lambda^{\prime} \subset V \subset A \\
\text { connected and } \\
\text { simply connected }}} N^{\prime} \sup _{1 \leqq j \leqq N^{\prime}} \mu_{V}^{h^{*}, k+1}\left\{m_{S_{j}}(\varphi) \leqq \frac{3}{8}\right\} \\
& \leqq N^{\prime} e^{-\frac{4}{9} \beta \frac{5}{8} N^{\prime}} \leqq N e^{-\frac{1}{4} \beta N} \leqq e^{-\frac{1}{5} \beta N} .
\end{aligned}
$$

We now complete the proof with an estimate of the last term in (3.8). The basic observation here is that if $\varphi \in Y^{c}$, then there is a contour $\alpha$ for $\varphi$ touching both $\delta \Lambda$ and $\delta \Lambda^{\prime}$, which implies

$$
\left|\alpha_{0}\right|=|\alpha \backslash \delta \Lambda| \geqq 2 d\left(\delta \Lambda, \delta \Lambda^{\prime}\right) \geqq 2\lfloor N / 20\rfloor .
$$

It is easy to check that for any dual edge $e^{*}$,

$$
\#\left\{\alpha \in C_{B}(\Lambda): \alpha \ni e^{*},\left|\alpha_{0}\right|=l\right\} \leqq K^{l+4 N+1},
$$

where $K^{l}$ is an upper bound for the number of $\alpha$ of length $l$ containing a fixed edge. Then, by Lemma 3.2, setting $l_{0}=2\lfloor N / 20\rfloor$,

$$
\begin{aligned}
\mu_{\Lambda}^{h^{*}, \emptyset}\left(Y^{c}\right) & \leqq \sum_{\substack{\alpha \in C_{B}(\Lambda) \\
\alpha \cap \delta \Lambda \neq \emptyset,\left|\alpha_{0}\right| \geqq l_{0}}} \mu_{\Lambda}^{h^{*}, \emptyset}\{\alpha \text { is a contour for } \varphi\} \\
& \leqq \sum_{e^{*} \in \delta \Lambda} \sum_{\substack{\alpha \in C_{B}(\Lambda) \\
\alpha \ni e^{*},\left|\alpha_{0}\right| \geqq l_{0}}} \mu_{\Lambda}^{h^{*}, \emptyset}\{\alpha \text { is a contour for } \varphi\} \\
& \leqq|\delta \Lambda| \sum_{l=l_{0}}^{\infty} K^{l+4 N+1} e^{-0.99 \beta l} \leqq 2 K^{l_{0}+4 N+1} e^{-0.99 \beta l_{0}}
\end{aligned}
$$

Together with (3.7), (3.8) and (3.9) this proves the theorem (if $N \geqq 1000$ then $\left.l_{0} \geqq \frac{1}{11} N\right)$. 
3.2. Bound on the variance of $f_{0}$. In order to find a lower bound on the variance of $f_{0}$, we prove

Lemma 3.4. Let $\beta$ be large enough, $1 \leqq k \leqq k_{\max }$. Take $\Lambda=Q_{N}$ with $N>$ $10 / h_{k}^{*}(\beta)$. Then

$$
\begin{aligned}
& \mu_{\Lambda}^{h_{k}^{*}(\beta), \emptyset}\left\{\varphi \in \Omega_{\Lambda}: M_{\Lambda}(\sigma(\varphi))<0\right\} \geqq e^{-\frac{1}{20} \beta N}, \\
& \mu_{\Lambda}^{h_{k}^{*}(\beta), \emptyset}\left\{\varphi \in \Omega_{\Lambda}: M_{\Lambda}(\sigma(\varphi))>0\right\} \geqq e^{-\frac{1}{20} \beta N} .
\end{aligned}
$$

Proof. Let $h^{*}=h_{k}^{*}(\beta)$. We assume, for simplicity that $N=2 L+1$ is odd and subdivide $\Lambda$ into two rectangles, so we consider the central horizontal row

$$
R=\{[-L, L] \cap \mathbb{Z}\} \times\{0\} .
$$

Then we write

$$
\Lambda=\Lambda_{1} \cup R \cup \Lambda_{2},
$$

where $\Lambda_{1}$ is the $N \times L$ rectangle above $R$ and $\Lambda_{2}$ is the rectangle below $R$. Consider now the events

$$
\begin{aligned}
& X=\left\{\varphi \in \Omega_{\Lambda}: \varphi(x)=k, \forall x \in R\right\}, \\
& Y_{i}=\left\{\varphi \in \Omega_{\Lambda}: M_{\Lambda_{i}}(\sigma)<0\right\} \quad i=1,2 .
\end{aligned}
$$

Then

$$
\begin{aligned}
\mu_{\Lambda}^{h^{*}, \emptyset}\left\{M_{\Lambda}(\sigma)<0\right\} & \geqq \mu_{\Lambda}^{h^{*}, \emptyset}\left(Y_{1} \cap Y_{2} \mid X\right) \mu_{\Lambda}^{h^{*}, \emptyset}(X) \\
& =\mu_{\Lambda_{1}}^{J, h^{*}, k}\left(Y_{1}\right) \mu_{\Lambda_{2}}^{J, h^{*}, k}\left(Y_{2}\right) \mu_{\Lambda}^{h^{*}, \emptyset}(X),
\end{aligned}
$$

where $J$ is taken as in (3.3). To take care of the first two terms, we observe that $H\left(\Lambda_{1}, \frac{1}{4}\right)$ holds for $J$ by an argument very similar to the proof of statement $(*)$ in Lemma 3.2. So, using the row decomposition as in (3.9), and Corollary 4.3 of [CM], we get

$$
\mu_{\Lambda_{1}}^{J, h^{*}, k}\left(Y_{1}\right)=1-\mu_{\Lambda_{1}}^{J, h^{*}, k}\left(Y_{1}^{c}\right) \geqq 1-L e^{-\frac{1}{9} \beta N} \geqq \frac{1}{2}
$$

(same for $Y_{2}$ ).

Let's consider now the last term in (3.10). If $\varphi \in X$ then

$$
H_{\Lambda}^{h^{*}, \emptyset}(\varphi)=H_{\Lambda_{1}}^{J, h^{*}, k}(\varphi)+H_{\Lambda_{2}}^{J, h^{*}, k}(\varphi)+h^{*} k N
$$

so

$$
\mu_{\Lambda}^{h^{*}, \emptyset}(X)=\frac{\sum_{\varphi \in X} e^{-\beta H_{\Lambda}^{h^{*}, \emptyset}(\varphi)}}{Z^{h^{*}, \emptyset}(\Lambda)}=\frac{Z^{J, h^{*}, k}\left(\Lambda_{1}\right) Z^{J, h^{*}, k}\left(\Lambda_{2}\right)}{Z^{h^{*}, \emptyset}(\Lambda)} e^{-\beta h^{*} k N} .
$$

The idea is again to use the cluster expansion to estimate the quotient of partition functions. For this purpose we introduce a small coupling on the boundary of $\Lambda$,

$$
\bar{J}(x, y)=\left\{\begin{array}{ll}
1 & \text { if }[x, y]^{*} \notin \delta \Lambda \\
10 \zeta & \text { if }[x, y]^{*} \in \delta \Lambda
\end{array},\right.
$$


where we have set $\zeta=1000^{-1}$. Jensen inequality (see Proposition 4.5 of [CM]) and Proposition 3.5 (which is given below) yield

$$
\frac{Z^{\bar{J}, h^{*}, k}(\Lambda)}{Z^{h^{*}, \emptyset}(\Lambda)} \geqq \exp \left[-10 \zeta \beta|\delta \Lambda| \sup _{x \in \partial \Lambda} \mathbf{E}_{\Lambda}^{h^{*}, \emptyset}|\varphi(x)-k|\right] \geqq e^{-11 \zeta \beta|\delta \Lambda|} .
$$

Hence, since $H\left(\Lambda_{1}, \frac{1}{4}\right), H\left(\Lambda_{2}, \frac{1}{4}\right)$ holds for $J$, and $H(\Lambda, 10 \zeta)$ holds for $\bar{J}$ for all $V \subset \subset \mathbb{Z}^{2}$, all three partition functions

$$
Z^{J, h^{*}, k}\left(\Lambda_{1}\right), \quad Z^{J, h^{*}, k}\left(\Lambda_{2}\right), \quad Z^{\bar{J}, h^{*}, k}(\Lambda)
$$

have a cluster expansion, thanks to Theorem 4.1 of [CM]. With the help of Theorem 4.4 of $[\mathrm{CM}]$ it is not difficult to show that

$$
\frac{Z^{J, h^{*}, k}\left(\Lambda_{1}\right) Z^{J, h^{*}, k}\left(\Lambda_{2}\right)}{Z^{\bar{J}, h^{*}, k}(\Lambda)} \geqq e^{\beta h^{*} k N-\varepsilon(\beta) N},
$$

where $\varepsilon(\beta) \rightarrow 0$ when $\beta \rightarrow \infty$. Together with (3.12),(3.14) this implies

$$
\mu_{\Lambda}^{h^{*}, \emptyset}(X) \geqq e^{-45 \zeta \beta N},
$$

and then, by $(3.10),(3.11)$

$$
\mu_{\Lambda}^{h^{*}, \emptyset}\left\{M_{\Lambda}(\sigma)<0\right\} \geqq e^{-50 \zeta \beta N} .
$$

The proof of the second statement is identical.

In the next proposition we complete the proof, by showing how to get the second inequality in (3.14). We will actually give a more general statement than the one needed.

Proposition 3.5. Let $\beta$ be large enough, $1 \leqq k \leqq k_{\max }, \Lambda=Q_{N}, N>10 / h_{k}^{*}(\beta)$. Then

$$
\sup _{x \in \Lambda} \mathbf{E}_{\Lambda}^{h_{k}^{*}(\beta), \emptyset}|\varphi(x)-k| \leqq 1.1 .
$$

Proof. Let $h^{*}=h_{k}^{*}(\beta)$ and for any positive integer $k$ let, as in [CM],

$$
\begin{gathered}
I_{k}(\beta)=\left[h_{k}^{-}(\beta), h_{k}^{+}(\beta)\right], \quad h_{k}^{-}(\beta)=\frac{4}{\beta} e^{-4 \beta k}, \\
h_{k}^{+}(\beta)=\frac{1}{4 \beta} e^{-4 \beta(k-1)}, h_{1}^{+}=\frac{1}{\beta} e^{-\frac{\beta}{25} J} .
\end{gathered}
$$

By FKG we can write

$$
\begin{aligned}
& \mu_{\Lambda}^{h^{*}, \emptyset}\{\varphi(x) \geqq n\} \leqq \mu_{V}^{h, \emptyset}\{\varphi(x) \geqq n\} \quad \forall h \in I_{k+1}(\beta), \\
& \mu_{\Lambda}^{h^{*}, \emptyset}\{\varphi(x) \leqq n\} \leqq \mu_{V}^{h, \emptyset}\{\varphi(x) \leqq n\} \quad \forall h \in I_{k}(\beta) .
\end{aligned}
$$

Choose now $h \in I_{k+1}(\beta)$, and a positive integer $j$. We want to show that the RHS of (3.16) is less than $e^{-\frac{\zeta}{3} \beta j}$ when $n=k+1+j$. As in [CM], for $V \subset \Lambda$, we define 
the events

$$
S_{+}(\Lambda, j, V)=\left\{\varphi \in \Omega_{\Lambda}: \begin{array}{l}
\text { there exists a path }\left(x_{1}, \ldots, x_{s}\right) \text { such that } \\
x_{1} \in V, x_{s} \in \partial \Lambda \text { and } \varphi\left(x_{i}\right) \geqq j \text { for each } i
\end{array}\right\} .
$$

If $x \in \Lambda$ and $j>0$, trivially we have that $\varphi(x) \geqq k+j+1$ if and only if $\varphi \in$ $S_{+}(\Lambda, k+1+j,\{x\})$. Thus, by Theorem 5.2 of $[\mathrm{CM}]$ we get

$$
\mu_{V}^{h, \emptyset}\{\varphi(x) \geqq k+1+j\} \leqq e^{-\zeta \beta j} .
$$

If, on the contrary, $x \in \Lambda \backslash \partial \Lambda$, then we define the event $F$ as

$$
F=S_{+}\left(\Lambda, k+1+\lfloor j / 2\rfloor+1, \partial^{+}\{x\}\right) \text {. }
$$

So

$$
\mu_{\Lambda}^{h, \emptyset}\{\varphi(x) \geqq k+1+j\} \leqq \mu_{\Lambda}^{h, \emptyset}\left(\varphi(x) \geqq k+1+j \mid F^{c}\right)+\mu_{\Lambda}^{h, \emptyset}(F) .
$$

By Proposition A1.1 of [CM] one finds

$$
\mu_{\Lambda}^{h, \emptyset}\left(\varphi(x) \geqq k+1+j \mid F^{c}\right) \leqq \sup _{\substack{V \ni x \\ V \text { connected and } \\ \text { simply connected }}} \mu_{V}^{h, k+1+\lfloor j / 2\rfloor}\{\varphi(x) \geqq k+1+j\} .
$$

Using FKG again and Proposition 3.5 of [CM], and letting $m=\lfloor j / 2\rfloor$, we get

$$
\begin{aligned}
\mu_{V}^{h, k+1+m}\{\varphi(x) \geqq k+1+j\} & \leqq \mu_{V}^{h, k+1+m}(\varphi(x) \geqq k+1+j \mid \varphi(y)>m \forall y \in V) \\
& =\mu_{V}^{h, k+1}\{\varphi(x) \geqq k+1+j-m\} \leqq e^{-\frac{1}{5} \beta(j-m)} \\
& \leqq e^{-\frac{1}{10} \beta j} .
\end{aligned}
$$

On the other side Theorem 5.2 of [CM] says that

$$
\mu_{\Lambda}^{h, \emptyset}(F) \leqq e^{-\zeta \beta(1+m)} \leqq e^{-\frac{1}{2} \zeta \beta j}
$$

These last two inequalities, together with (3.16), (3.18) give

$$
\mu_{\Lambda}^{h^{*}, \emptyset}\{\varphi(x) \geqq k+1+j\} \leqq e^{-\frac{\zeta}{3} \beta j} .
$$

In a similar way one finds, for $j>0$

$$
\mu_{\Lambda}^{h^{*}, \emptyset}\{\varphi(x) \leqq k-j\} \leqq e^{-\frac{\zeta}{3} \beta j}
$$

Therefore, for all $x \in \Lambda$,

$$
\mathbf{E}_{\Lambda}^{h^{*}, \emptyset}|\varphi(x)-k| \leqq 1+2 \sum_{j=1}^{\infty} e^{-\frac{\zeta}{3} \beta j}+\leqq 1.1
$$

\section{Lower Bound on the Gap at $h_{k}^{*}(\beta)$}

In this section we complete the proof of Theorem 1.1 by proving a lower bound on the gap of the generator of the dynamics for $h=h_{k}^{*}(\beta)$ and arbitrary boundary 
conditions, which is of the same order of the upper bound discussed in the previous section, namely a negative exponential of the boundary of the square $Q_{L}$.

As in [CM] we define $N_{0}(h)=\left(\zeta^{3} h\right)^{-1}$, where $\zeta=1000^{-1}$. We prove that (remember that we always assume $0 \leqq J(x, y) \leqq 1$ and that $J \in \delta Q$ has been defined in Sect. 1.2)

Theorem 4.1. If $\beta$ is large enough, then, for each $1 \leqq k \leqq k_{\max }=\left\lfloor e^{\frac{\beta \zeta}{20}}\right\rfloor$, there exists a positive constant $G\left(\beta, h_{k}^{*}(\beta)\right)$ such that

$$
\inf _{J \in \delta Q} \inf _{\psi \in \Omega} \operatorname{gap}\left(L_{Q}^{J, h_{k}^{*}(\beta), \psi}\right) \geqq G\left(\beta, h_{k}^{*}(\beta)\right)^{\log L} e^{-80 \beta k L}
$$

for any square $Q$ of side $L \geqq 20 N_{0}\left(h_{k}^{*}(\beta)\right)$.

Remarks. (1) Free boundary conditions are recovered by simply setting $J(x, y)=1$ everywhere but $J(x, y)=0$ for all $x, y$ such that $[x, y]^{*} \in \delta Q$. Moreover, using the fact that the gap is positive uniformly in the boundary conditions for each $L \geqq\lfloor 8 / h+1\rfloor$ (Proposition 2.2), we can easily find a constant $C_{1}(\beta, h)>0$ such that statement (ii) of Theorem 1.1 holds.

(2) By using a more sophisticated approach which requires the so-called "surgery" technique one can replace the factor $80 \beta k$ with $80 \beta$.

Proof. Let $h^{*}=h_{k}^{*}(\beta), N_{0}=N_{0}\left(h^{*}\right)$ and $\hat{N}_{0}=10 N_{0}$. The proof is obtained by recursion as follows. For $L \in \mathbb{Z}_{+}$, let

$$
g_{L}(\beta, h)=\inf _{J \in \delta Q_{L}} \inf _{\psi \in \Omega} \operatorname{gap}\left(L_{Q_{L}}^{J, h, \psi}\right) .
$$

Proposition 2.2 says that $g_{L}>0$ if $L \geqq\lfloor 8 / h+1\rfloor$. Given now $L \geqq 2 \hat{N}_{0}$, it is possible to find positive integers $s,\left\{L_{i}\right\}_{i=1}^{s}$ such that

$$
\begin{aligned}
& L=L_{0}>L_{1}>\cdots>L_{s}=\hat{N}_{0}, \\
& \frac{5}{4} L_{i} \leqq L_{i-1} \leqq \frac{7}{4} L_{i} \quad i=1, \ldots, s
\end{aligned}
$$

(a proof of this is given in Appendix A2). We clearly have

$$
s \leqq \frac{\log L-\log \hat{N}_{0}}{\log (5 / 4)} .
$$

Then we prove the following recursive inequality

$$
g_{L_{i-1}}\left(\beta, h^{*}\right) \geqq g_{L_{i}}\left(\beta, h^{*}\right) G_{0}\left(\beta, h^{*}\right) e^{-20 \beta k L_{i}} \quad \forall 1 \leqq i \leqq s
$$

for a suitable $G_{0}\left(\beta, h^{*}\right)>0$. Iterating (4.1) and observing that

$$
\sum_{i=1}^{s} L_{i} \leqq L \sum_{i=1}^{s}(4 / 5)^{i} \leqq 4 L
$$


we get

$$
g_{L}\left(\beta, h^{*}\right) \geqq g_{\hat{N}_{0}}\left(\beta, h^{*}\right) \prod_{i=1}^{s}\left[G_{0}\left(\beta, h^{*}\right) e^{-20 \beta k L_{i}}\right] \geqq G\left(\beta, h^{*}\right)^{\log L} e^{-80 \beta k L}
$$

for some $G\left(\beta, h^{*}\right)>0$, and so the theorem is proven.

In order to establish (4.1), we actually prove the slightly more general inequality

$$
\inf _{\frac{5}{4} N \leqq L \leqq \frac{7}{4} N} g_{L}\left(\beta, h^{*}\right) \geqq g_{N}\left(\beta, h^{*}\right) G_{0}\left(\beta, h^{*}\right) e^{-20 \beta k N} \quad \forall N \geqq \hat{N}_{0} .
$$

We proceed as follows. Let $N \geqq \hat{N}_{0}, 5 N / 4<L \leqq 7 N / 4$ and $\Lambda=Q_{L}$. $\Lambda$ can be obtained in a unique way (apart from permutations) as a union of four overlapping squares of side $N$ that we denote by $\Lambda_{i}, i=1, \ldots, 4$ (see Fig. 1 ). Let $\Lambda_{1}$ be the northeast square in $\Lambda, \Lambda_{2}$ the northwest one and so on, proceeding counterclockwise. Let $\mathscr{D}=\left\{\Lambda_{1}, \Lambda_{2}, \Lambda_{3}, \Lambda_{4}\right\}$, and consider the block-dynamics defined in Appendix A1, determined by $\beta, h^{*}, J$, the b.c. $\psi$ and the collection $\mathscr{D}$, through the Markov generator $L_{\mathscr{D}}^{J, h^{*}, \psi}$ given by (A1.1).

Thanks to Proposition A1.1 we know that

$$
\operatorname{gap}\left(L_{\Lambda}^{J, h^{*}, \psi}\right) \geqq \operatorname{gap}\left(L_{\mathscr{D}}^{J, h^{*}, \psi}\right) g_{N}\left(\beta, h^{*}\right) .
$$

Thus, in order to prove the theorem, it remains to show that there exists $G_{0}\left(\beta, h^{*}\right)>0$ such that

$$
\inf _{J \in \delta \Lambda} \inf _{\psi \in \Omega} \operatorname{gap}\left(L_{\mathscr{D}}^{J, h^{*}, \psi}\right) \geqq G_{0}\left(\beta, h^{*}\right) e^{-20 \beta k N} .
$$

By Proposition A1.2, this in turn follows, if we can prove that

$$
\begin{gathered}
\mathbb{P}_{\Lambda}^{J, h^{*}, \psi,(\varphi, \bar{\varphi})}\left\{\left(\varphi_{t}, \bar{\varphi}_{t}\right): \varphi_{1}=\bar{\varphi}_{1}\right\} \geqq G_{0}\left(\beta, h^{*}\right) e^{-20 \beta k N} \quad \text { for all } \varphi, \bar{\varphi} \\
\text { such that } \varphi \leqq \bar{\varphi},
\end{gathered}
$$

where $\left(\varphi_{t}, \bar{\varphi}_{t}\right)$ is the coupled process defined in Appendix A1. The idea at this point is that in order to have $\varphi_{t}=\bar{\varphi}_{t}$ on $\Lambda$ it is "almost" sufficient to impose that the two configurations agree on a subset of $\Lambda$ whose cardinality is of the order of the boundary. Let in fact (see Fig. 1)

$$
A_{i}=\Lambda_{i} \cap\left(\bigcup_{j \neq i} \partial^{+} \Lambda_{j}\right) \quad i=1, \ldots, 4 .
$$

Using the explicit construction and notation for this coupled process given in Appendix A1, we have

$$
\mathbb{P}_{\Lambda}^{J, h^{*}, \psi,(\varphi, \bar{\varphi})}\left\{\varphi_{1}=\bar{\varphi}_{1}\right\} \geqq p_{1} p_{2} p_{3},
$$

where $p_{1}$ is the probability of having $\tau(6) \leqq 1, p_{2}$ is the probability that the order in which the first six blocks are updated is given by

$$
l(1), \ldots, l(6)=1,3,2,4,1,3 J,
$$

and $p_{3}$ is the probability of having $\varphi_{\tau(6)}=\bar{\varphi}_{\tau(6)}$, given (4.6). Define now the events (the random variables $(\eta, \bar{\eta})$ have been introduced in Appendix A1)

$$
F_{i, j}^{\left(\varphi^{\prime}, \varphi^{\prime \prime}\right)}=\left\{(\eta(x), \bar{\eta}(x))_{i, j}^{\left(\varphi^{\prime}, \varphi^{\prime \prime}\right)}=(1,1) \forall x \in A_{i}\right\} .
$$


Then we observe, and this is the key remark, that, since the interaction is between nearest neighbors, if the events $F_{1,1}^{\left(\varphi_{0}, \bar{\varphi}_{0}\right)}$ and $F_{3,2}^{\left(\varphi_{\tau(1)}, \bar{\varphi}_{\tau(1)}\right)}$ happen, then necessarily (given (4.6)) $\varphi_{\tau(6)}=\bar{\varphi}_{\tau(6)}$. Thus, taking into account

(i) that $(\eta(x), \bar{\eta}(x))=(1,1)$ if and only if $\bar{\eta}(x)=1$, get

(ii) the properties of the standard coupling $v_{\Lambda_{i}}^{\varphi^{\prime}, \varphi^{\prime \prime}}(\eta, \bar{\eta})$ given in Sect. 1.3, we

$$
\begin{aligned}
p_{3} & \geqq \inf _{\varphi^{\prime} \leqq \varphi^{\prime \prime}, \tilde{\varphi}^{\prime} \leqq \tilde{\varphi}^{\prime \prime}} \operatorname{Prob}\left(F_{1,1}^{\left(\varphi^{\prime}, \varphi^{\prime \prime}\right)} \cap F_{3,2}^{\left(\tilde{\varphi}^{\prime}, \tilde{\varphi}^{\prime \prime}\right)}\right) \\
& \geqq \inf _{J \in \delta \Lambda_{1}} \inf _{\varphi^{\prime} \in \Omega}\left(\mu_{\Lambda_{1}}^{J, h^{*}, \varphi^{\prime}}\left\{\varphi \in \Omega_{\Lambda_{1}}: \varphi(x)=1, \forall x \in A_{1}\right\}\right)^{2} .
\end{aligned}
$$

Clearly the event $\left\{\varphi(x)=1 \forall x \in A_{i}\right\}$ is a decreasing event so that, using the FKG property we get

$$
\mu_{\Lambda_{1}}^{J, h^{*}, \varphi^{\prime}}\left\{\varphi(x)=1 \forall x \in A_{1}\right\} \geqq \prod_{x \in A_{1}} \mu_{\Lambda_{1}}^{J, h^{*}, \varphi^{\prime}}\{\varphi(x)=1\} .
$$

Now we subdivide the $x$ 's in $A_{1}$ into two groups, depending on whether they are close to the boundary or not. Let then

$$
\begin{aligned}
& A_{1}^{\prime}=\left\{x \in A_{1}: d\left(x, \partial \Lambda_{1}\right) \leqq N_{0}\right\}, \\
& A_{1}^{\prime \prime}=A_{1} \backslash A_{1}^{\prime} .
\end{aligned}
$$

We claim that

(a) $\left|A_{1}^{\prime}\right| \leqq 4 N_{0}$ and $\left|A_{1}^{\prime \prime}\right| \leqq 2 N$.

(b) If $x \in A_{1}^{\prime}$, then for all $J \in \delta \Lambda_{1}, \varphi^{\prime} \in \Omega, \mu_{\Lambda_{1}}^{J, h^{*}, \varphi^{\prime}}\{\varphi(x)=1\} \geqq b_{2}\left(b, h^{*}\right)>0$.

(c) If $x \in A_{1}^{\prime \prime}$, then for all $J \in \delta \Lambda_{1}, \varphi^{\prime} \in \Omega, \mu_{\Lambda_{1}}^{J, h^{*}, \varphi^{\prime}}\{\varphi(x)=1\} \geqq \frac{1}{8} e^{-4 \beta k}$.

(a), (b) and (c) together with the obvious bound

$$
p_{1} p_{2} \geqq e^{-\beta}
$$

clearly imply (4.3) with

$$
G_{0}\left(\beta, h^{*}\right)=e^{-\beta} b_{2}\left(\beta, h^{*}\right)^{8 N_{0}}
$$

and so the theorem would be proven.

- To prove (a) it is sufficient to observe that $A_{1}=T_{v} \cup T_{h}$ (see Fig. 1), where $T_{v}$ is a vertical line of length $N$ whose distance from the vertical sides of $\Lambda_{1}$ is greater than

$$
((L-N) \wedge(2 N-L))-2 \geqq \frac{1}{5} N \geqq 2 N_{0},
$$

since $5 N / 4<L \leqq 7 N / 4$. An analogous statement holds for $T_{h}$.

- (b) is proven in Proposition 3.2 of [CM].

- The proof of (c) is a little more involved and we have to use the fact that at distances greater than $N_{0}$ from the boundary it is very likely to have $k \leqq \varphi \leqq k+1$. Let in fact $x \in A_{1}^{\prime \prime}$. Let $V_{0}$ be a square of side $N_{0}$ (or $N_{0}+1$ if $N_{0}$ is even) centered 
at $x$ and let $V_{1}$ be the square still centered at $x$ but with side $N_{0}-2\left\lfloor N_{0} / 4\right\rfloor-2$. By (1.7), taking into account that if $J \in \delta \Lambda_{1}$ then $J(x, y)=1$ for all $\{x, y\} \subset \Lambda_{1}$, we obtain

$$
\inf _{J \in \delta \Lambda_{1}} \inf _{\varphi^{\prime} \in \Omega} \mu_{\Lambda_{1}}^{J, h^{*}, \varphi^{\prime}}\{\varphi(x)=1\} \geqq \inf _{\varphi^{\prime} \in \Omega} \mu_{V_{0}}^{h^{*}, \varphi^{\prime}}\{\varphi(x)=1\} .
$$

Take now $\bar{h}=\frac{1}{4 \beta} e^{-4 \beta k} \leqq h^{*}$. Because $\{\varphi(x)=1\}$ is a negative event, by the FKG property,

$$
\mu_{V_{0}}^{h^{*}, \varphi^{\prime}}\{\varphi(x)=1\} \geqq \mu_{V_{0}}^{\bar{h}, \varphi^{\prime}}\{\varphi(x)=1\} .
$$

Furthermore, if we let

$$
A=S_{+}\left(V_{0}, k+2, \partial^{+} V_{1}\right)
$$

(the event $S_{+}(\Lambda, j, V)$ has been defined in Proposition 3.5) by 5.1 of $[\mathrm{CM}]$ and A1.1 of $[\mathrm{CM}]$ we have

$$
\begin{aligned}
\mu_{V_{0}}^{\bar{h}, \varphi^{\prime}}\{\varphi(x)=1\} & \geqq \mu_{V_{0}}^{\bar{h}, \varphi^{\prime}}\left(\varphi(x)=1 \mid A^{c}\right) \mu_{V_{0}}^{\bar{h}, \varphi^{\prime}}\left(A^{c}\right) \\
& \geqq \frac{1}{2} \sup _{\substack{V: V_{1} \subset V \subset V_{0} \\
V \text { connected and } \\
\text { simply connected }}} \mu_{V}^{\bar{h}, k+1}\{\varphi(x)=1\} .
\end{aligned}
$$

On the other side, letting

$$
F=\left\{\varphi \in \Omega_{V}: \varphi(y)=k+1 \forall y \in V, \text { such that }|x-y|=1\right\},
$$

and using Proposition 3.5 of [CM], we find, for each simply connected $V$ containing $V_{1}$,

$$
\begin{aligned}
& \mu_{V}^{\bar{h}, k+1}\{\varphi(x)=1\} \mu_{V}^{\bar{h}, k+1}(\varphi(x)=1 \mid F) \mu_{V}^{\bar{h}, k+1}(F) \\
& \quad \geqq \mu_{V}^{\bar{h}, k+1}(\varphi(x)=1 \mid F)\left(1-4 \sup _{y \in V} \mu_{V}^{\bar{h}, k+1}\{\varphi(y) \neq k+1\}\right) \\
& \quad \geqq \frac{1}{2} \mu_{\{x\}}^{\bar{h}, k+1}\{\varphi(x)=1\} .
\end{aligned}
$$

But an explicit computation, gives

$$
\mu_{\{x\}}^{\bar{h}, k+1}\{\varphi(x)=1\} \geqq \frac{1}{2} e^{-4 \beta k} .
$$

In this way we have found

$$
\inf _{J \in \delta \Lambda_{1}} \inf _{\varphi^{\prime} \in \Omega} \mu_{\Lambda_{1}}^{J, h^{*}, \varphi^{\prime}}\{\varphi(x)=1\} \geqq \frac{1}{8} e^{-4 \beta k} \quad \forall x \in A_{1}^{\prime \prime} .
$$

Acknowledgements. This work started while one of the authors (F.M) was profiting from a very stimulating research period at the Newton Institute in Cambridge in the framework of the special semester dedicated to "Spatial Structure of Random Fields." He would like to thank the organizers for the kind invitation and the opportunity to discuss with several specialists in the field. We would like also to thank Mazel for interesting discussions about their work. 


\section{Appendix A1. The Block Dynamics}

\section{Given}

(i) the hamiltonian (1.1), with parameters $(\beta, h, J)$,

(ii) a boundary condition $\psi \in \Omega$,

(iii) a collection $\mathscr{D}=\left\{\Lambda_{1}, \ldots, \Lambda_{n}\right\}$ of finite (possibly overlapping) subsets of $\mathbb{Z}^{2}$, and letting

$$
\Lambda=\bigcup_{i=1}^{n} \Lambda_{i}
$$

we define as the standard block dynamics (with respect to $\beta, h, J, \psi, \mathscr{D}$ ) the Markov process on $\Omega_{\Lambda}$ whose generator is

$\left(L_{\mathscr{D}}^{\psi} f\right)(\varphi)=\sum_{i=1}^{n} \sum_{\eta \in \Omega} \mu_{\Lambda_{i}}^{\varphi}(\eta)[f(\eta)-f(\varphi)]$ for all $\varphi$ such that $\varphi_{\Lambda^{c}}=\psi_{\Lambda^{c}}$

The sum over $\eta$ is actually restricted to those configurations that agree with $\varphi$ outside $\Lambda_{i}$ because of (1.6). A straightforward computation shows that $L_{\mathscr{D}}^{\psi}$ is still a bounded self-adjoint operator on $L^{2}\left(\Omega_{\Lambda}, d \mu_{\Lambda}^{\psi}\right)$. We assume to have chosen $\beta, h, J$ and won't mention them explicitly in the following. Then

Proposition A1.1. For all $\beta, h>0$, all $J, \psi$, let $L_{\Lambda}^{\psi}$ be given by (1.8) with transition rates satisfying $\left(\mathrm{H}_{1}\right)-\left(\mathrm{H}_{5}\right)$. Then

$$
\operatorname{gap}\left(L_{\Lambda}^{\psi}\right) \geqq \operatorname{gap}\left(L_{\mathscr{D}}^{\psi}\right) \inf _{i} \inf _{\varphi \in \Omega} \operatorname{gap}\left(L_{\Lambda_{i}}^{\varphi}\right)\left(\sup _{x \in \Lambda} \#\left\{i: \Lambda_{i} \ni x\right\}\right)^{-1} .
$$

Proof. Let

$$
g=\inf _{i} \inf _{\varphi \in \Omega} \operatorname{gap}\left(L_{\Lambda_{l}}^{\varphi}\right)
$$

Thanks to (1.12), (A1.2) is proven if we can show that

$$
\mathscr{E}_{\mathscr{D}}^{\psi}(f, f) \leqq g^{-1} \sup _{x \in \Lambda} \#\left\{i: \Lambda_{i} \ni x\right\} \mathscr{E}_{\Lambda}^{\psi}(f, f) \quad \text { for all } f \in L^{2}\left(\Omega_{\Lambda}, d \mu_{\Lambda}^{\psi}\right)
$$

But, using (1.7), we find

$$
\begin{aligned}
\mathscr{E}_{\mathscr{D}}^{\psi}(f, f) & \leqq \frac{1}{2} \sum_{\varphi \in \Omega} \mu_{\Lambda}^{\psi}(\varphi) \sum_{i} \sum_{\eta \in \Omega} \mu_{\Lambda_{i}}^{\varphi}(\eta)[f(\eta)-f(\varphi)]^{2} \\
& =\frac{1}{2} \sum_{\varphi \in \Omega} \sum_{\varphi^{\prime} \in \Omega} \mu_{\Lambda}^{\psi}\left(\varphi^{\prime}\right) \sum_{i} \mu_{\Lambda_{l}}^{\varphi^{\prime}}(\varphi) \sum_{\eta \in \Omega} \mu_{\Lambda_{i}}^{\varphi}(\eta)[f(\eta)-f(\varphi)]^{2} \\
& =\sum_{\varphi^{\prime} \in \Omega} \mu_{\Lambda}^{\psi}\left(\varphi^{\prime}\right) \sum_{i} \operatorname{Var}_{\Lambda_{i}}^{\varphi^{\prime}}(f) \leqq g^{-1} \sum_{\varphi^{\prime} \in \Omega} \mu_{\Lambda}^{\psi}\left(\varphi^{\prime}\right) \sum_{i} \mathscr{E}_{\Lambda_{i}}^{\varphi^{\prime}}(f, f) .
\end{aligned}
$$


On the other side, by (1.7) again,

$$
\begin{aligned}
& \sum_{\varphi^{\prime} \in \Omega} \mu_{\Lambda}^{\psi}\left(\varphi^{\prime}\right) \sum_{i} \mathscr{E}_{\Lambda_{i}}^{\varphi^{\prime}}(f, f) \\
& \quad=\frac{1}{2} \sum_{\varphi^{\prime} \in \Omega} \mu_{\Lambda}^{\psi}\left(\varphi^{\prime}\right) \sum_{i} \sum_{\varphi \in \Omega} \mu_{\Lambda_{i}}^{\varphi^{\prime}}(\varphi) \sum_{x \in \Lambda_{l}, s= \pm 1} c(x, \varphi, s)\left[f\left(\varphi^{x, s}\right)-f(\varphi)\right]^{2} \\
& \quad \leqq \sup _{x \in \Lambda} \#\left\{i: \Lambda_{i} \ni x\right\} \mathscr{E}_{\Lambda}^{\psi}(f, f) .
\end{aligned}
$$

(A1.3) and the proposition are thus proven.

We want to introduce now a coupling between two copies of the block dynamic process in such a way to preserve the order given by the initial conditions. Given $\psi, \varphi, \bar{\varphi}$ such that $\psi_{\Lambda^{c}}=\varphi_{\Lambda^{c}}=\bar{\varphi}_{\Lambda^{c}}$, consider the process $\left(\varphi_{t}, \bar{\varphi}_{t}\right)$ which starts from $(\varphi, \bar{\varphi})$ at time zero and evolves according to the generator

$$
\left(\hat{L}_{\mathscr{D}}^{\psi} f\right)(\varphi, \bar{\varphi})=\sum_{i} \sum_{\eta, \bar{\eta} \in \Omega} v_{\Lambda_{i}}^{\varphi, \bar{\varphi}}(\eta, \bar{\eta})[f(\eta, \bar{\eta})-f(\varphi, \bar{\varphi})],
$$

where $v_{\Lambda_{i}}^{\varphi, \bar{\varphi}}$ is the standard coupling defined in Sect. 1. Given the starting point $(\varphi, \bar{\varphi}) \in \Omega^{2}$, the process $\left(\varphi_{t}, \bar{\varphi}_{t}\right)$ can be constructed explicitly as follows. Let

(a) $\tau(j), j=1,2, \ldots$, be the jump times of a Poisson process with rate $n=$ number of blocks.

(b) $(\eta, \bar{\eta})_{i, j}^{(\varphi, \bar{\varphi})}$, for $(\varphi, \bar{\varphi}) \in \Omega^{2}, i=1, \ldots, n$ and $j=1,2, \ldots$ be a collection of $\Omega^{2}$ valued independent random variables (and independent of the $\tau_{j}$ 's) distributed according to $v_{\Lambda_{i}}^{\varphi, \bar{\varphi}}(\eta, \bar{\eta})$.

(c) $l(j)$ for $j=1,2, \ldots$ be a collection of i.i.d. (and independent of what has been defined before) random variables with uniform distribution on $\{1, \ldots, n\}$.

Define now

(1) $\left(\varphi_{0}, \bar{\varphi}_{0}\right)=(\varphi, \bar{\varphi})$,

(2) $\left(\varphi_{t}, \bar{\varphi}_{t}\right)=\left(\varphi_{\tau(j)}, \bar{\varphi}_{\tau(j)}\right)$, for all $\tau(j) \leqq t<\tau(j+1)$,

(2) $\left(\varphi_{\tau(j+1)}, \bar{\varphi}_{\tau(j+1)}\right)=(\eta, \bar{\eta})_{l(j+1), j+1}^{\left(\varphi_{\tau(j)}, \bar{\varphi}_{\tau(j)}\right)}$.

Then we have

Proposition A1.2. With respect to the quantities previously defined

(i) both $\varphi_{t}$ and $\bar{\varphi}_{t}$ have the same distribution as the process associated with the generator (A1.1).

(ii) If $\varphi \leqq \bar{\varphi}$, then

$$
\operatorname{Prob}\left\{\varphi_{t} \leqq \bar{\varphi}_{t}\right\}=1 \text { for all } t \geqq 0 .
$$

(iii) If $\operatorname{Prob}\left\{\varphi_{1}=\bar{\varphi}_{1}\right\} \geqq p$ for all starting points $\varphi, \bar{\varphi}$ such that $\varphi \leqq \bar{\varphi}$ (and such that they agree with $\psi$ on $\left.\Lambda^{c}\right)$, then

$$
\operatorname{gap}\left(L_{\mathscr{D}}^{\psi}\right) \geqq p .
$$

(i) is a standard computation. (ii) follows from the definition of $\left(\varphi_{t}, \bar{\varphi}_{t}\right)$ and from the properties of the coupling measure $v_{\Lambda}^{\varphi, \bar{\varphi}}$. To get (iii) we use the (time) Markov 
property which yields

$$
\operatorname{Prob}\left\{\varphi_{t} \neq \bar{\varphi}_{t}\right\} \leqq(1-p)^{\lfloor t\rfloor} \leqq e^{-(t-1) p} .
$$

\section{Appendix A2}

Proposition A2.1. Let $N \geqq 100, L \geqq 2 N$. Then there exist integers $s, L_{i}$, such that

$$
\begin{aligned}
& L=L_{0}>L_{1}>\cdots>L_{s}=N, \\
& \frac{5}{4} L_{i} \leqq L_{i-1} \leqq \frac{7}{4} L_{i} \quad i=1, \ldots, s .
\end{aligned}
$$

Proof. Let also $X$ be the set of $L$ 's such that the proposition holds. We want to show that $X$ contains all integers greater than or equal to $2 N$. Let $N_{0}^{+}=N_{0}^{-}=N$ and, for each $i \in \mathbb{Z}_{+}$, we set $K_{i}=\left[N_{i}^{-}, N_{i}^{+}\right]$, where

$$
N_{i}^{+}=\left\lfloor\frac{7}{4} N_{i-1}^{+}\right\rfloor \quad \text { and } \quad N_{i}^{-}=\left\lceil\frac{5}{4} N_{i-1}^{-}\right\rceil
$$

( $\lceil x\rceil$ is the smallest integer not less than $x$ ). Obviously

$$
K_{i} \supset \bigcup_{n \in K_{i-1}}[\lceil 5 n / 4\rceil,\lfloor 7 n / 4\rfloor]
$$

and, since for $n \geqq 100$,

$$
\lfloor 7 n / 4\rfloor \geqq\lceil 5(n+1) / 4\rceil,
$$

we have in fact

$$
K_{i}=\bigcup_{n \in K_{l-1}}[\lceil 5 n / 4\rceil,\lfloor 7 n / 4\rfloor] .
$$

So, for each $m \in K_{i}$ there exists $n \in K_{i-1}$ such that

$$
\frac{5}{4} \leqq \frac{m}{n} \leqq \frac{7}{4} \text {. }
$$

This implies

$$
X \supset \bigcup_{i=1}^{\infty} K_{i}
$$

Furthermore, since $N \geqq 100$, we have

$$
N_{2}^{-} \leqq(5 / 4)^{2} N+5 / 4+1 \leqq(7 / 4) N-1 \leqq N_{1}^{+},
$$

and it is easy to check, by induction, that

$$
N_{i}^{-} \leqq N_{i-1}^{+} \quad \forall i \geqq 2 .
$$

Thus we get

$$
\bigcup_{i=1}^{\infty} K_{i} \supset \bigcup_{i=2}^{\infty}\left[N_{i-1}^{+}, N_{i}^{+}\right]=\left[N_{1}^{+}, \infty\right) \supset[2 N, \infty) .
$$




\section{References}

[CM] Cesi, F., Martinelli, F.: On the layering transition of an SOS surface interacting with a wall I. Equilibrium results. Submitted to Commun. Math. Phys.

[DM] Dinaburg, E.I., Mazel, A.E.: Layering transition in SOS model with external magnetic field. J. Stat. Phys. 74, Nos 3/4, 533 (1994)

[DS] Dobrushin, R., Shlosman, S.: Constructive criterion for the uniqueness of Gibbs fields. Stat. Phys. and Dyn. Sys., Basel, Boston: Birkhäuser, 1985, pp. 371-403

[FP1] Fröhlich, J., Pfister, C.: Semi-infinite Ising model. I. Commun. Math. Phys. 109, 493 (1987)

[FP2] Fröhlich, J., Pfister, C.: Semi-infinite Ising model. II. Commun. Math. Phys. 112, 51 (1987)

[L] Ligget, T.M.: Interacting particles systems. Berlin, Heidelberg, NewYork: Springer, 1985

[LS] Lawler, G.F., Sokal, A.D.: Bounds on the $L^{2}$ spectrum for Markov chains and Markov processes: A generalization of Cheeger's inequality. Trans. Amer. Math. Soc. 309, No 2, 557 (1988)

[LY] Lu, S., Yau, H.T.: Spectral gap and logarithmic Sobolev inequality for Kawasaki and Glauber dynamics. Commun. Math. Phys. 156, 399 (1993)

[MO1] Martinelli, F., Olivieri, E.: Approach to equilibrium of Glauber dynamics in the one phase region I: The attractive case. Commun. Math. Phys. 161, 447 (1994)

[MO2] Martinelli, F., Olivieri, E.: Approach to equilibrium of Glauber dynamics in the one phase region II: The general case. Commun. Math. Phys. 161, 487 (1994)

[MOS] Martinelli, F., Olivieri, E., Schonmann, R.H.: For Gibbs state of 2D lattice spin systems weak mixing implies strong mixing. Commun. Math. Phys. 165, 33 (1994)

[S1] Schonmann, R.H.: Private communication

[S2] Schonmann, R.H.: Second order large deviation estimates for ferromagnetic systems in the phase coexistence region. Commun. Math. Phys. 112, 409 (1987)

[SSh] Schonmann, R.H., Shlosman, S.B.: Complete analiticity for 2D Ising model completed. Preprint (1994)

[SZ] Stroock, D.W., Zegarlinski, B.: The logarithmic Sobolev inequality for discrete spin on a lattice. Commun. Math. Phys. 149, 175 (1992)

Communicated by J.L. Lebowitz 
